\title{
Producing filler-gap dependencies: structural priming evidence for two distinct combinatorial processes in production
}

\author{
Shota Momma \\ University of Massachusetts, Amherst
}

\begin{abstract}
A previous model of long-distance dependency production claims that speakers use two distinct pieces of structures containing clause-taking verbs like believe and the complementizer that when planning sentences with cross-clausal fillergap dependencies (e.g., Who did the breeder believe (that) the dog bit?) vs. when planning sentences without (e.g., The breeder believed (that) the dog bit them.). Under a certain assumption about the lexical boost effect, this model predicts that the lexical boost effect for that-priming occurs only when prime and target sentences both contain a cross-clausal filler-gap dependency or when neither does. In the current study, a computational model of structural priming implementing the core claims of the previous filler-gap dependency production model was built to show that this prediction coherently follows from the model. The prediction of the model was then tested in five recall-based structural priming experiments. Speakers showed a larger complementizer priming effect when prime and target sentences share a clause-taking verb (i.e., the lexical boost effect). But the lexical boost effect was selective to when both prime and target sentences contained a cross-clausal filler-gap dependency (Experiment 3) and when neither did (Experiment 1). Critically, the lexical boost effect was absent when only either prime or target sentences contained a filler-gap dependency crossing the complementizer structure (Experiments 2, 4, and 5), confirming the
\end{abstract}

\footnotetext{
Data, scripts, and materials used in this study are available here: https://osf.io/us4wg/?view only=5a11ac1033c94473a988263427b31119.
} 
prediction of the model.

\section{Introduction}

Grammatical relationships can hold at an arbitrarily long distance. For instance, in English wh-questions such as who does the girl think the boy met?, who forms closer syntactic and semantic relationships with the linearly further verb met than with the closer verb think. This is often referred to as filler-gap dependencies where the filler (e.g., who) is the phrase that fills the gap, the missing object position of the transitive verb, met. Filler-gap dependencies are an apparent exception to the otherwise general rule that grammatical relations occur locally, usually within a clause. Given their exceptional status, they have offered excellent clues about the nature and limit of computational mechanisms in grammar (Chomsky, 1957, 1965, 1973, 1986, 1981, 1995; Pollard \& Sag, 1994; Kroch \& Joshi, 1985; Frank, 2004; Ross, 1967) and processing (Fodor, 1978; Frazier et al., 1983; Frazier \& Flores d'Arcais, 1989; Garnsey et al., 1989; Aoshima et al., 2004; Omaki et al., 2015; Clifton \& Frazier, 1989; Wanner \& Maratsos, 1978). In comparison, filler-gap dependencies have received minimal attention in sentence production. Existing models of sentence production (Levelt, 1989; Kempen \& Hoenkamp, 1987; Bock \& Levelt, 1994; Ferreira et al., 2018; Chang et al., 2006; Bock \& Ferreira, 2013; Griffin \& Ferreira, 2006) rarely discuss how speakers plan filler-gap dependencies. However, precisely because of the exceptional status of filler-gap dependencies, understanding their production is likely to be critical in understanding structure-building mechanisms in production.

Against this background, Momma (2021) recently proposed a model of fillergap dependency production based on Tree-Adjoining Grammar (Joshi et al., 1975; Kroch \& Joshi, 1985; Frank, 2004). Although this model focused on capturing the time-course of filler-gap dependency formation processes in production, under certain assumptions, it makes an untested prediction about structural priming (Levelt \& Kelter, 1982; Bock, 1986), the tendency for speakers to re-use structures they have recently encountered, specifically the structural 
priming of English complementizers (Ferreira, 2003). Ferreira (2003) showed that after encountering sentences like the girl thought that the boy met the cat, speakers are more likely to use that in the sentence they subsequently produce, than after encountering sentences without that like the girl thought the boy met the cat. The model claims that clause-taking verbs (such as think, say, argue, etc.) head two distinct pieces of trees containing the complementizer structure depending on whether target sentences involve cross-clausal filler-gap dependencies. That is, the model claims that the planning of sentences like who does the girl think that the boy met? and the girl thinks that the boy met someone involves two distinct pieces of structures containing a complementizer and a clause-taking verb. Under the assumption that the complementizer priming found by Ferreira (2003) can be boosted by repeating such distinct pieces of structures, the model makes a prediction that repeating a clause-taking verb between prime and target sentences enhances the magnitude of the complementizer priming effect, but only when both prime and target sentences contain cross-clausal filler-gap dependencies or when neither does. The primary purpose of the current study is to show that this prediction indeed follows from the core set of assumptions in Momma (2021) via a simple computational model and to test this prediction experimentally. Because this prediction does not follow from any other models of structural priming (Chang et al., 2006; Reitter et al., 2011; Pickering \& Branigan, 1998) or sentence production more generally (Bock \& Levelt, 1994; Bock \& Ferreira, 2013; Levelt, 1989), the confirmation of this prediction would give a new insight on structural building in speaking.

\subsection{Structural priming and lexical boost effects}

Numerous studies have shown that speakers tend to reuse the structures that they have recently encountered, a phenomenon known as structural priming (Levelt \& Kelter, 1982; Bock, 1986). For example, in English, after encountering a double object (DO) structure like in the girl gave the boy the book, speakers are more likely to use the DO structure in the subsequent production, as in the student handed the teacher his essay than after encountering a prepositional 
dative (PD) structure like in the girl gave the book to the boy. The structural priming effect has been observed in many languages (e.g., English: Bock (1986); Griffin \& Weinstein-Tull (2003); Pickering \& Branigan (1998); Bock \& Griffin (2000); German: Chang et al. (2015); Melinger \& Dobel (2005); Dutch: Levelt \& Kelter (1982); Hartsuiker \& Kolk (1998); American Sign Language: Hall et al. (2015); Mandarin Chinese: Cai et al. (2012); among others languages) with many different alternations (see Pickering \& Ferreira (2008) for an overview and Mahowald et al. (2016) for a meta-analysis, and Pickering \& Branigan (2019) for overview on structural priming in less-studied languages). An important property of structural priming is that prime and target sentences do not need to share any words, possibly except for passive priming, where the repetition of by may be necessary (Ziegler et al., 2019). This suggests that primed structures are at least partially independent of words in speakers mind.

Although structural priming can be observed without repeating any words, many previous studies have also shown that the size of the structural priming effect is bigger when content words (but not function words; Bock (1989)), especially the word that heads the primed structures, are repeated between prime and target sentences. For example, the size of the structural priming effect from the girl showed the boy a bicycle to the student showed the teacher an essay is larger than from the girl gave the boy a bicycle. This is known as the lexical boost effect (Pickering \& Branigan, 1998).

The lexical boost effect has been shown across different alternations, including in the active/passive alternation (Messenger et al., 2012), the double object/prepositional object dative alternation (Pickering \& Branigan, 1998), and the word-order alternation in the particle verb construction (Konopka \& Bock, 2009). Although the lexical boost effect often results from the repetition of verbs that head primed structures, some studies (Cleland \& Pickering, 2003) showed that repeating nominal heads also increases the priming effect of pre-nominal vs. post-nominal adjectival modification (e.g., the green table vs. the table that is green). Often, the lexical boost effect is taken as the evidence for some privileged connection between abstract structural representations (dubbed combinatorial 
nodes, Pickering \& Branigan (1998)) and lexical representations, which can be enhanced when a relevant structure and a connected word co-occur. Note that, contrary to this interpretation, Scheepers et al. (2017) reported that repeating any content words between prime and target sentences triggers the lexical boost effect and that increasing the number of shared words increases the magnitude of the lexical boost effect near-linearly. It was claimed that the lexical boost effect should not be used as evidence for lexically specific structural representations or some privileged relationship between a repeated word and a primed structure. However, more recent evidence suggests that the lexical boost effect can only be observed when the head of a primed structure is repeated (Carminati et al., 2019), and that the lexical boost effect from non-head words is observed only when prime sentences were visible during target production (van Gompel et al., 2022). Thus, we take the lexical boost effect to be reasonable evidence for a privileged relationship between a repeated word and a primed structure. See General Discussion for a more detailed discussion on this issue.

The most relevant type of structural priming in the current study is the priming of English complementizers, reported by Ferreira (2003). Using a memorybased production task, Ferreira (2003) showed that speakers were more likely to produce that in target sentences like the teacher noticed (that) the failing student skipped class given prime sentences with that like the director announced that Hollywoods hottest actor would be playing the part than given minimally different prime sentences without that. This that-priming effect was argued to be syntactic because the demonstrative that (as in that book) or the that in complex noun phrases (as in the claim that) do not prime the complementizer that, and because the absence, not just the presence, of that can be primed compared to a neutral baseline.

To my knowledge, the lexical boost effect for that-priming has not been shown experimentally, but there are various reasons to believe that it should be observed. For example, Roland et al. (2006) found that the identity of clause-taking verbs is a strong predictor of whether that is present. That is, the complementizer choice shows a clear verb bias, just like dative structures (Gahl 
et al., 2004; Van de Velde et al., 2015; Bernolet \& Hartsuiker, 2010; Garnsey et al., 1997). Given the verb bias of complementizer choice, the presence or absence of that may plausibly be encoded in the lemma representations of clausetaking verbs. This possibility is naturally compatible with the linguistic analysis that the presence or absence of that is conditioned by verbs. For example, it has been argued that the null complementizer is less acceptable if the relevant clause is not strictly subcategorized by a verb (Stowell (1981); see Bianchi \& Frascarelli (2017) for a recent overview). Given those views, if the lexical boost effect arises from the priming of some link between the lemma of a repeated word and a primed structure (e.g., Pickering \& Branigan (1998)), the lexical boost effect for that-priming is naturally expected.

The issue of whether the that-priming can be lexically boosted is theoretically interesting in its own right. However, the primary purpose of the current study is not just to test if the that-priming can be lexically boosted but rather to test the prediction of a TAG-based filler-gap dependency production model proposed by Momma (2021) using the that-priming effect. Thus, I now explain how Tree-Adjoining Grammar (TAG) theories (Kroch \& Joshi, 1985; Joshi \& Schabes, 1997; Joshi et al., 1975) usually handle filler-gap dependencies, the essence of the TAG-based model by Momma (2021), and how it, under a certain assumption about the lexical boost effect, makes a prediction about when the lexical boost effect should and should not be observed for that-priming.

\subsection{Filler-gap dependencies in Tree Adjoining Grammar}

Building on previous proposals by Ferreira (2000) and Frank (2004), Momma (2021) proposed a model of filler-gap dependency production based on the grammatical formalism known as Tree-Adjoining Grammar (TAG; Kroch \& Joshi (1985); Joshi \& Schabes (1997); Joshi et al. (1975)). To explain the model proposed by Momma (2021), a basic introduction of TAG has to be provided first.

Basic building blocks of sentences in TAG are already complex objects with internal hierarchical structures, known as elementary trees. TAG derives even 
more complex objects, called derived trees, by combining elementary trees. There are primarily two ways to combine elementary trees: substitution and adjoining. Substitution replaces (rewrites) an open frontier (foot) node, usually argument slots (like subject or object slots) with another elementary tree whose root node is category-matching with the relevant substitution site. For instance, an elementary tree headed by a transitive verb like chase has two open frontier Determiner Phrase (DP) nodes, which can be replaced by substituting DP elementary trees headed by $\operatorname{dog}$ and cat to yield The dog chases the cat (Figure 1). Conventionally, a node that can be substituted is indicated by a downward arrow.

Figure 1: An example TAG derivation of a simple transitive sentence the dog chases the cat (adopted with slight modification from Momma (2021)). CP $=$ Complementizer Phrase, IP $=$ Inflectional phrase, $V P=$ Verb Phrase, $D P=$ Determiner Phrase. $t=$ traces that are coreferential with the DP with the same index. In generative theories, CPs are phrases headed by complementizers, which introduces a clause. IPs are phrases headed by auxiliary verbs and tense and agreement morphemes.

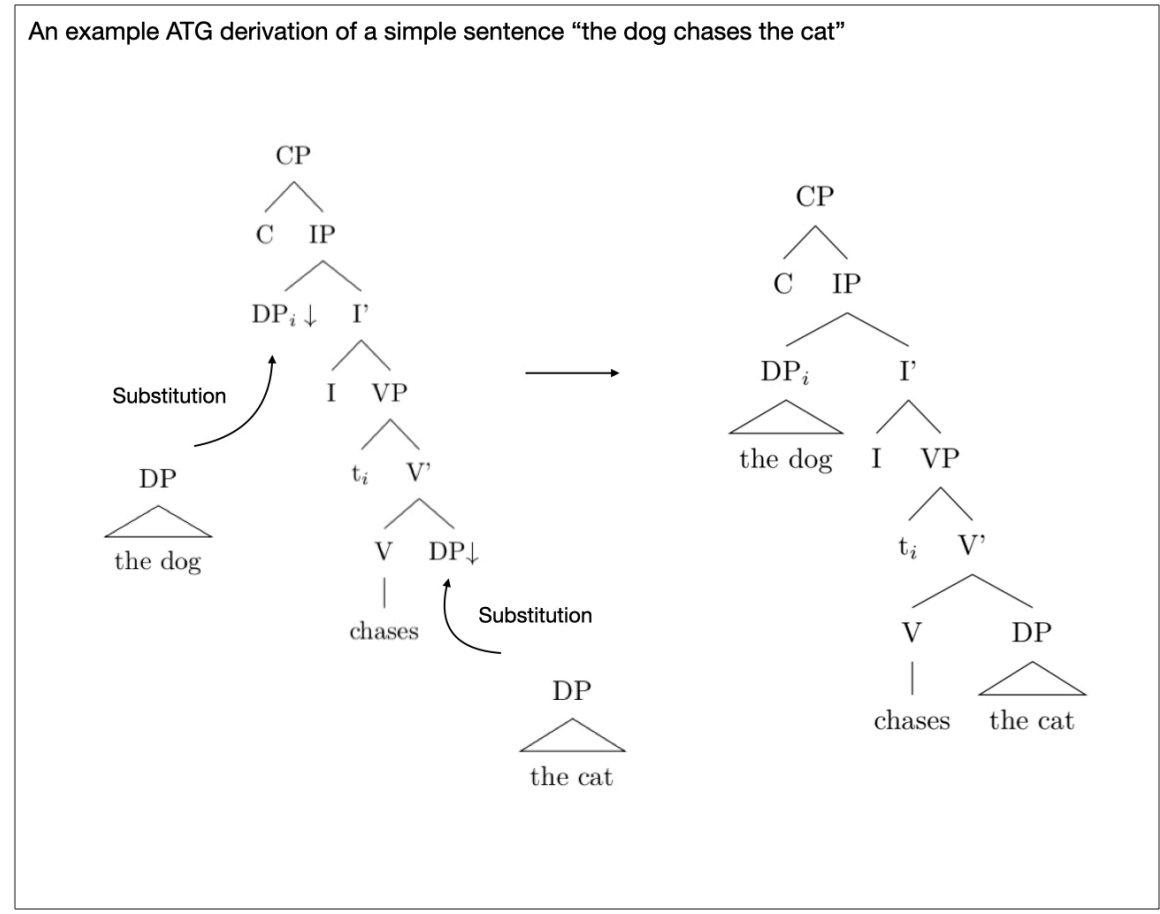


Adjoining replaces any node with a special type of elementary trees termed auxiliary trees. By definition, the top and one of the frontier nodes of auxiliary trees must have the identical category, meaning that auxiliary trees contain a recursive piece of phrase structure. Adjoining is used to introduce recursive elements, such as adverbs and adjectives (among other things). For example, the adverb often can be introduced to the derived tree the dog chases the cat via adjoining to yield the dog often chases the cat (Figure 2).

Figure 2: An example of adjoining in TAG (adopted from Momma (2021)).

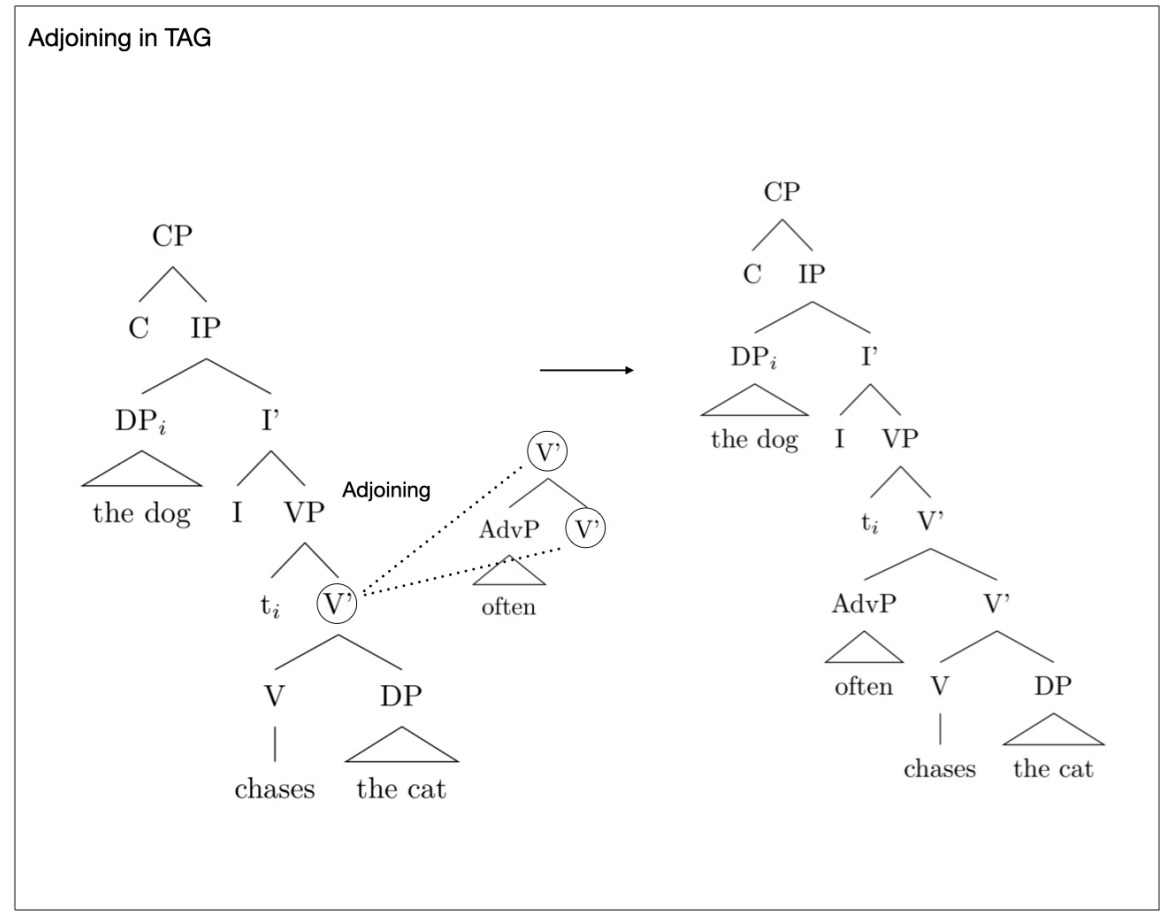

The size of elementary trees can in principle be arbitrarily large or small, but for a TAG theory to be restrictive, there must be some constraints on the size of elementary trees. The following hypothesis captures the common essence of various approaches (Frank, 2004).

- The fundamental TAG hypothesis: Every syntactic dependency is ex- 
pressed locally within a single elementary tree.

This hypothesis imposes substantive restrictions on the size of elementary trees. On the one hand, elementary trees must be big enough to contain syntactic dependencies. On the other hand, they must be small enough to capture the locality of syntactic dependencies. Most relevantly for current purposes, any filler-gap dependency must be contained in a single elementary tree, according to the fundamental TAG hypothesis. However, filler-gap dependencies span an indefinite distance (in principle). That is, there must be elementary trees that contain the filler and the gap but not material in between (Frank, 2004; Kroch \& Joshi, 1985). The insertion of material in between the filler and the gap is done via adjoining, as in Figure 3. In the left-most tree in Figure 3, the filler and the gap are simultaneously contained in a single elementary tree (the leftmost tree in Figure 3). That is, any filler-gap dependency is initially local. The auxiliary tree headed by think is then adjoined to the C' node of the elementary tree containing the filler-gap dependency. This adjoining operation expands the initially local filler-gap dependency, making it cross-clausal.

For current purposes, the $\mathrm{C}$ node, $\mathrm{C}$ ' node, and $\mathrm{CP}$ nodes are all assumed to carry some features encoding the presence or absence of that, as shown in Figure 3. Notice that the C' node of the elementary tree containing a filler-gap dependency simultaneously carries two conflicting features: [null] and [that] (written as [null/that] in Figure 3). This is an extension of the idea proposed by Vijay-Shanker (1992) adopted also in Frank (2004). In their system, the C' node of an elementary tree containing a cross-clausal filler-gap dependency is simultaneously a part of matrix and embedded clauses, and it has conflicting $[+\mathrm{Q}$ (uestion)/-Q] features. This conflicting feature expresses the idea that the matrix clause is a question, but the embedded clause is not. This conflicting feature makes adjoining of an auxiliary tree headed by a verb like think obligatory. This obligatoriness is good because adjoining is indeed necessary to yield a grammatical string when generating sentences containing cross-clausal filler-gap dependencies (e.g., Who that the dog chases? is not a grammatical string, so 
do you think must be added via adjoining). Importing this idea, in addition to the $[+\mathrm{Q} /-\mathrm{Q}]$ feature, the $\mathrm{C}$ ' node is assumed to carry two features encoding the complementizer type of the matrix and embedded clauses simultaneously. The C' node can have only the feature encoding the null complementizer when the embedded clause is without that. In that case, the conflicting $[+\mathrm{Q} /-\mathrm{Q}]$ feature (although not explicitly written in Figure 3) still ensures that adjoining takes place.

Figure 3: An example of TAG adjoining expanding a initially local filler-gap dependency.

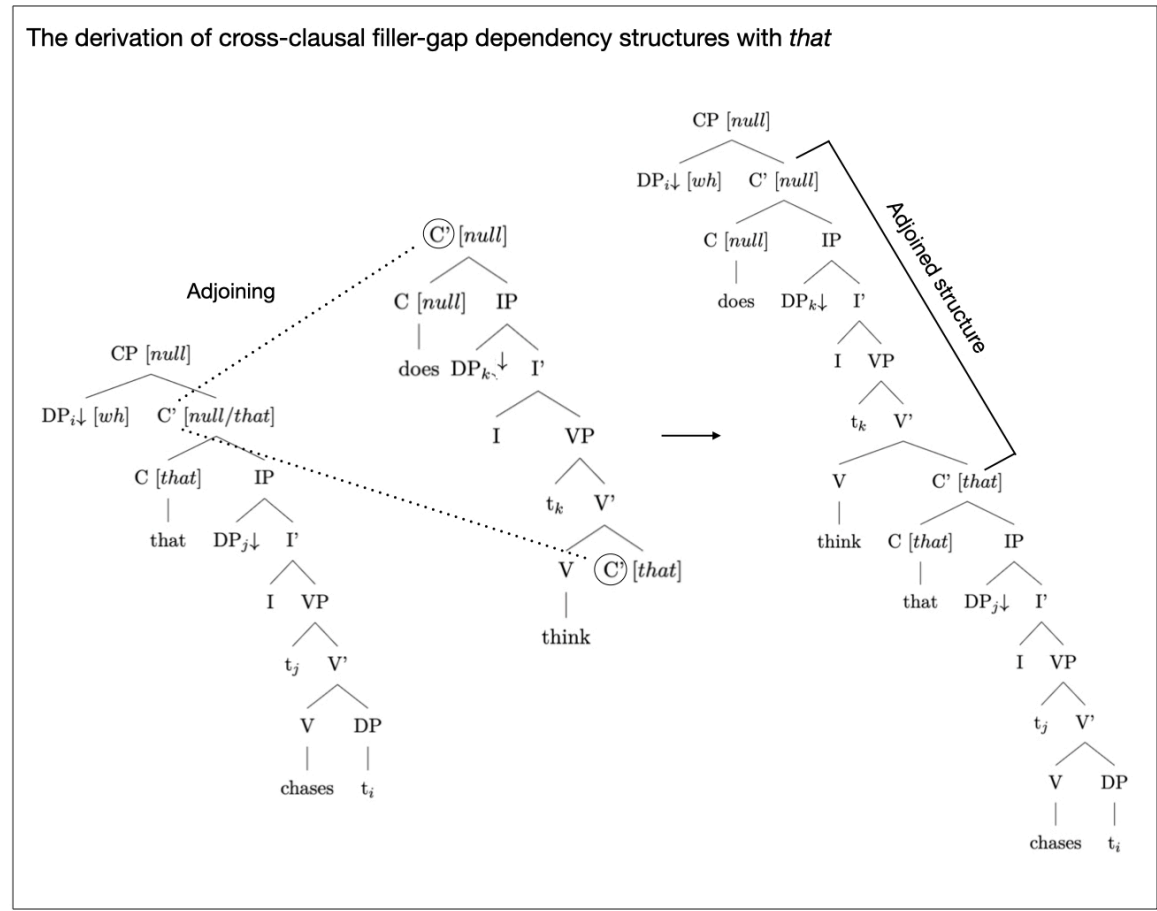

Importantly, in Frank (2004), complement clauses in sentences without crossclausal filler-gap dependencies are composed with clause-taking verbs like think via substitution. ${ }^{1}$ As shown in Figure 4, the elementary tree headed by think is a

\footnotetext{
${ }^{1}$ Unless the embedded verb also takes the same type of complement clause that the matrix verb took, in which case the system can use either substitution or adjoining for the same
} 
non-auxiliary tree with a $\mathrm{CP}$ argument slot, and a CP-rooted elementary tree is substituted to that slot to yield a bi-clausal structure. Thus, in the TAG system such as Frank (2004), there are two distinct elementary trees headed by verbs like think depending on whether there is a cross-clausal filler-gap dependency. This means that structural representations headed by clause-taking verbs like think are distinct when there is a cross-clausal filler-gap dependency vs. when there is not. For comparison, in Figure 5, two elementary trees are shown side by side.

Figure 4: An example of TAG derivation of bi-clausal structures with no cross-clausal filler-gap dependencies

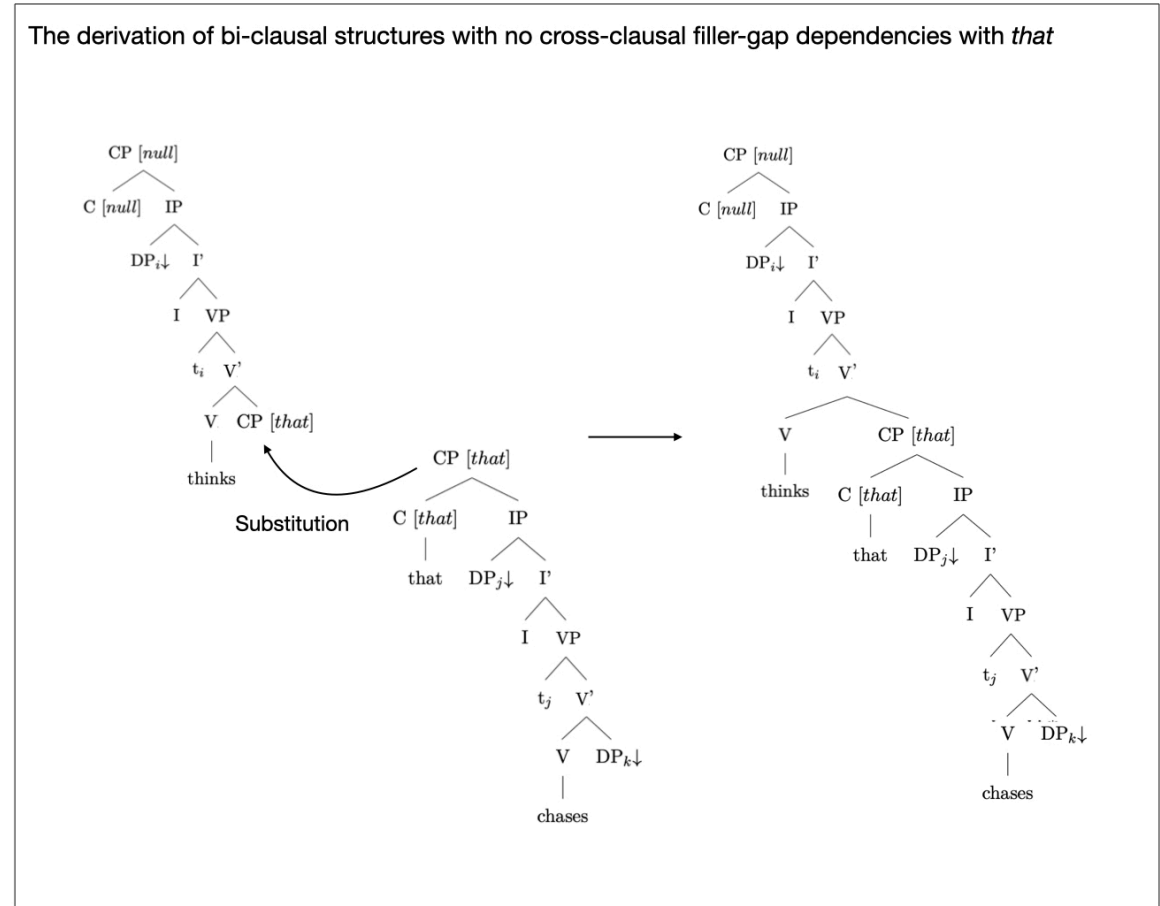

Turning to the model of production, Momma (2021) proposed that elementary trees correspond to planning domains in production and that the result. 
Figure 5: The non-auxiliary tree headed by think for sentences without a cross-clausal fillergap dependency (left) and the auxiliary tree headed by think for sentences with a cross-clausal filler-gap dependency (right).

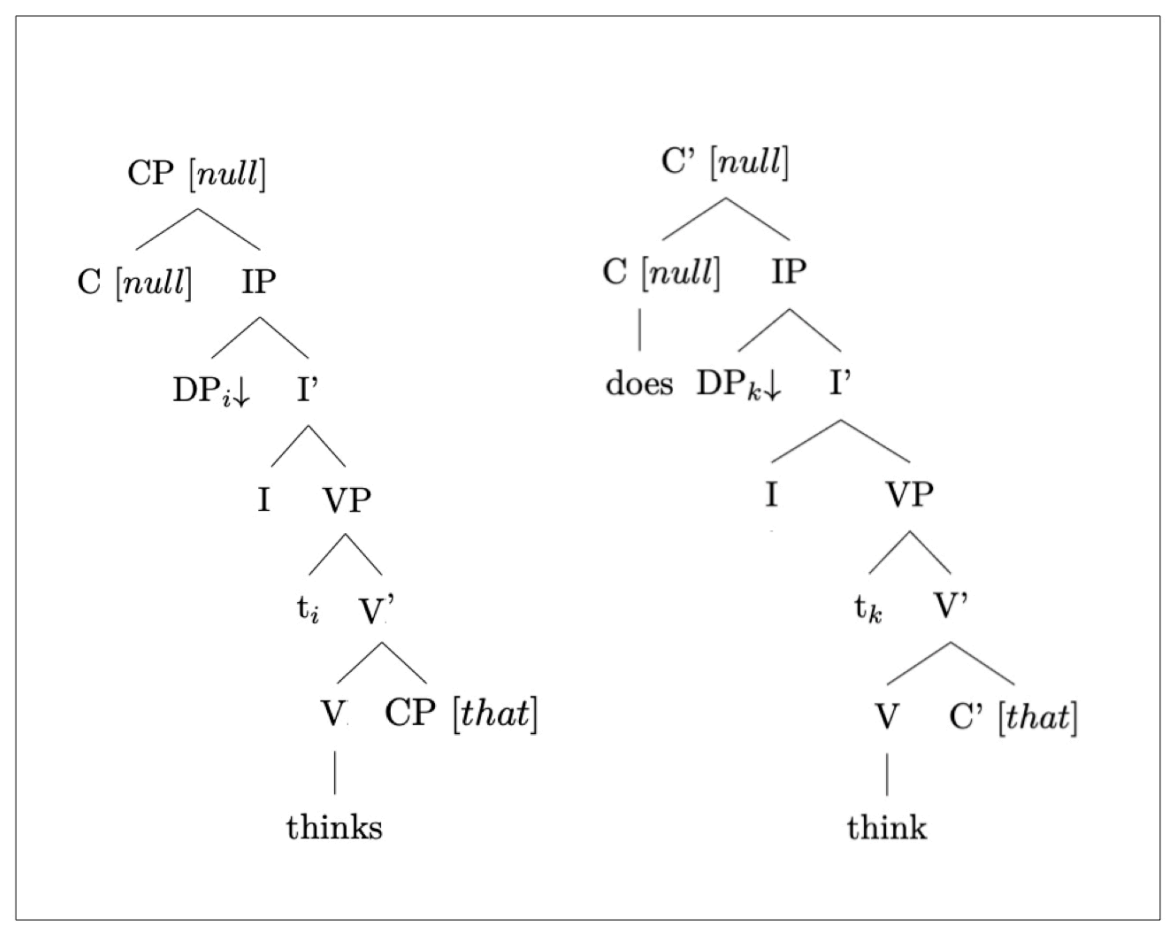

derivational procedures of TAG transparently reflect structure-building processes in production, adopting the version of TAG theories developed by Frank (2004) mostly as it was proposed (see also Ferreira (2000)). Thus, in Momma (2021), cross-clausal filler-gap dependency production involves the processing stage where the filler and the gap are represented simultaneously without the material in between them, just like how TAG derives cross-clausal filler-gap dependencies via adjoining. The subjects of matrix clauses as well as clause-taking verbs are adjoined to the structure containing filler-gap dependencies later in the process, just like how TAG derivation creates cross-clausal filler-gap dependencies. Momma (2021) offered some evidence suggesting that speakers indeed plan the gap structure before starting to speak the filler and before planning 
the lemma of the matrix subject. Because this model formulates cross-clausal filler-gap dependencies using the production analogue of adjoining in TAG, it implicitly, but necessarily, claims that clause-taking verbs head two distinct elementary trees. The idea pursued in the current study is that those two distinct structures headed by verbs like think can be primed independently from each other.

\subsection{Structural priming in the TAG-based model}

Building on the representational and planning assumptions in Momma (2021), I propose a model in which structural priming arises from the following two sources: (a) strengthening of the connection between a concept and a node of an elementary tree and (b) residual activation of elementary trees. The lexically independent structural priming is accounted for by (a), and the lexical boost is accounted for by (b). Because this proposal assumes two sources of structural priming, it shares the essence of multi-component accounts of structural priming (e.g., Pickering \& Ferreira (2008); Hartsuiker et al. (2008); Jacobs et al. (2019)) and resembles the original proposal by Pickering \& Branigan (1998), although the representational assumptions and the details of the models are distinct.

To provide the proof-of-concept that the prediction follows from the core set of assumptions in the TAG-based model, I implemented two TAG-based models of structural priming in the form of a connectionist spreading-activation network (Collins \& Loftus (1975); Dell (1986); Roelofs (1992); Levelt et al. (1999); Roelofs (1997), among others), one for dative priming and the other for that-priming. These models are not meant to be a comprehensive model of sentence production, but they are simplified models that show that the TAG-

based models are compatible with basic structural priming effects and that they naturally derive the critical predictions the current experiments aim to test. The implementational details of those models are in Appendix A.

Figures 6 and 7 show the basic architecture of the models for capturing the dative priming effect and the that-priming effect, respectively. In both models, there are three levels of representation: the level at which conceptual 
representations are encoded (the concept level); the level at which the nodes of elementary trees are represented (the subtree level); and the level at which elementary trees are encoded (the elementary tree level). The subtree level can be thought of as the super-set of the lemma level in other production models, and the elementary tree level can be thought of as a higher-order level where complex structures (elementary trees) are represented. The relationship between the subtree level and the elementary tree level is essentially compositional; subtree level nodes are connected to elementary tree level nodes containing them. The information flows only in one direction, from the concept level to the subtree level and the elementary tree level, or from the concept level to the elementary tree level directly; there is no feedback activation flow. Mutually exclusive nodes at the subtree level are connected with bidirectional and symmetric inhibitory links. Because the subtree level is the level at which lemma representations reside, these inhibitory links embody the competitive nature of lemma selection (Levelt et al. (1999); Cutting \& Ferreira (1999); Roelofs (1992, 1997), but see Navarrete et al. (2014); Mahon et al. (2007)). Inhibitory links are not the only way to capture the competitive nature of lexical selection (Levelt et al., 1999; Roelofs, 1992), but here we adopt the view that lexical competition arises from lateral inhibition between lemmas (Cutting \& Ferreira (1999)). However, it should be noted that lateral inhibition at the subtree level is not strictly necessary to derive the predictions about structural priming.

In contrast to subtree level nodes, elementary tree level nodes are not connected with lateral inhibitory links. This is because previous studies have shown that having multiple structural options facilitates rather than inhibits production (Ferreira (1996); but see Hwang \& Kaiser (2014); Myachykov et al. (2013)). If lateral inhibitory links exist between different elementary trees, the model would predict the inhibitory effect when multiple structural options are available, contrary to the findings by Ferreira (1996). Thus, the current model aims to simultaneously capture the claim that lemma selection is competitive, but structure selection is not. However, again, it should be noted that this assumption is not strictly necessary to derive the critical predictions about structural 
priming.

Figure 6: A TAG-based model of the dative priming effect. T1 represents the elementary tree encoding the double object structure headed by give (see Figure 8). T2 represents the minimally different version of T1 where give is replaced with show. T3 represents the elementary tree encoding the prepositional dative structure with give, and T4 represents the minimally different version of T3 where give is replaced with show.

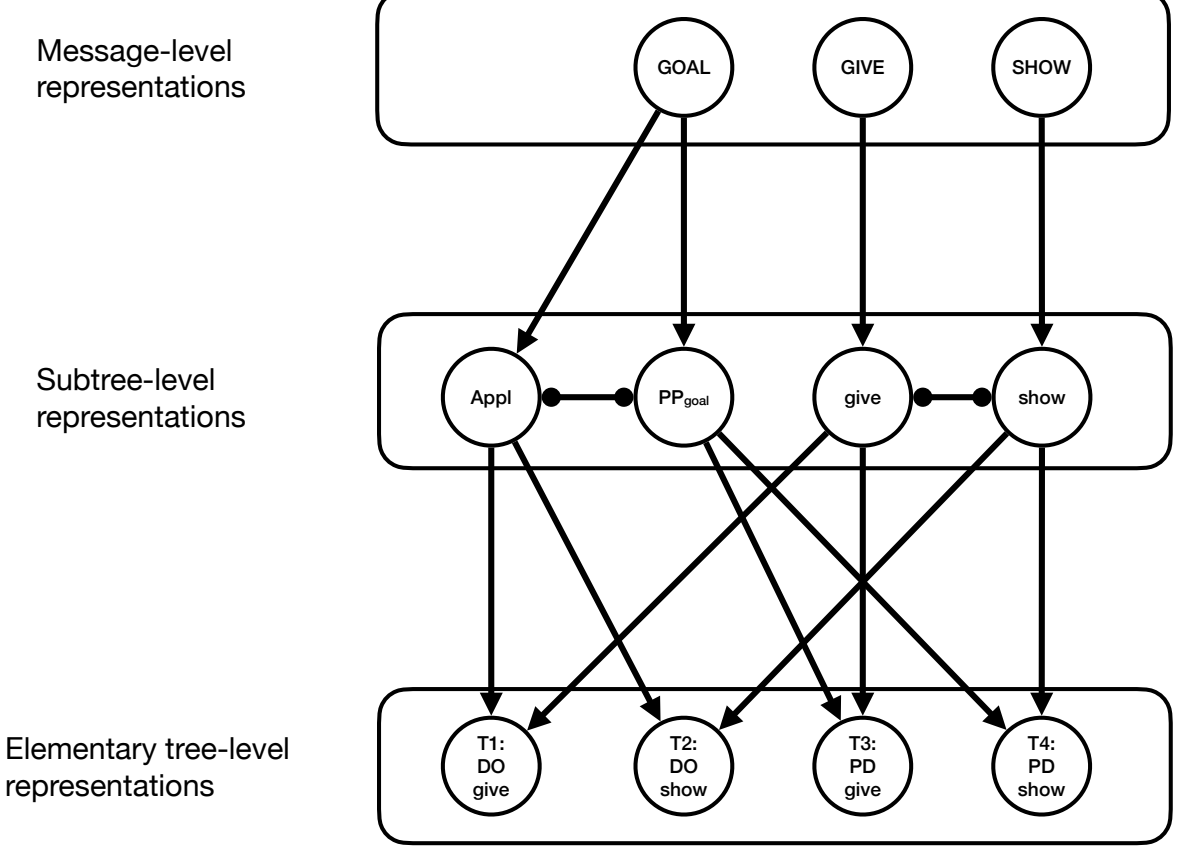

For the dative-priming model, following relatively modern theories of syntax in the generative tradition (e.g., Marantz (1993); Bruening (2001); Pylkkänen (2008) see Larson (2017)), I assume that the DO structure but not the PD structure involves a functional head known as the applicative head (henceforth $a p p l)$. Appl is assumed to assign the goal role to one of the objects in the double object construction (Figure 8). The PD structure is missing appl, but it instead contains the prepositional phrase structure associated with the goal role. This prepositional phrase structure is represented as $P P_{\text {goal }}$. Thus, the choice between the DO and the PD structures can be viewed as the choice between 
Figure 7: A TAG-based model of the that-priming effect. The nodes and connections in black are identically organized as in the dative priming model, and the nodes and connections in red are newly added parts of this model. T1 represents the elementary tree headed by think with that, T3 represents the minimally different version of $\mathrm{T} 1$ where the feature of that (on the CP node) is replaced with the feature of the null complementizer. T2 represents the minimally different version of $\mathrm{T} 1$ where the head verb think is replaced with say. T4 represents the minimally different version of $\mathrm{T} 2$ where the feature of that is replaced with the feature of the null complementizer. T5 represents the elementary tree encoding cross-clausal filler-gap dependency with that as shown in Figure 3. T6 represents the minimally different version of T5, where that is replaced with the null complementizer. A1 represents the auxiliary tree headed by think as shown in Figure 3. A2 represents the minimally different auxiliary tree of A1 where think is replaced by say. A3 and A4 represent the minimally different auxiliary trees of $\mathrm{A} 1$ and $\mathrm{A} 2$ respectively, where the feature of that in the foot node is replaced with the feature of the null complementizer.

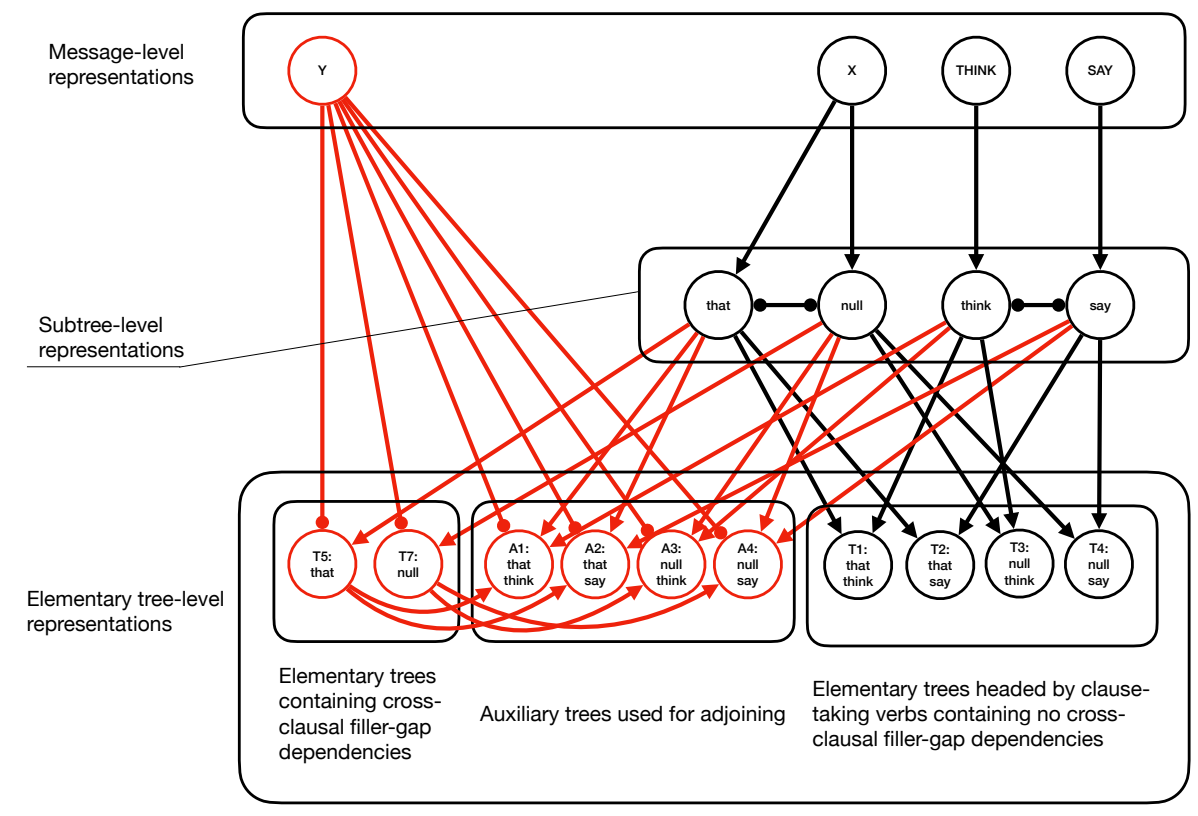

appl and the $P P_{\text {goal }}$ under the current approach. ${ }^{2}$

The dative priming model was designed as follows. Concept level nodes encode features that correspond to conceptual correlates of the goal role (represented as $G O A L$ ), and conceptual features associated with two verbs: give and show (represented as GIVE and SHOW, respectively). Here, the goal role is

\footnotetext{
${ }^{2}$ I owe the seed of this idea to Maxime Papillon.
} 
Figure 8: The structures of dative sentences assumed in the current study.

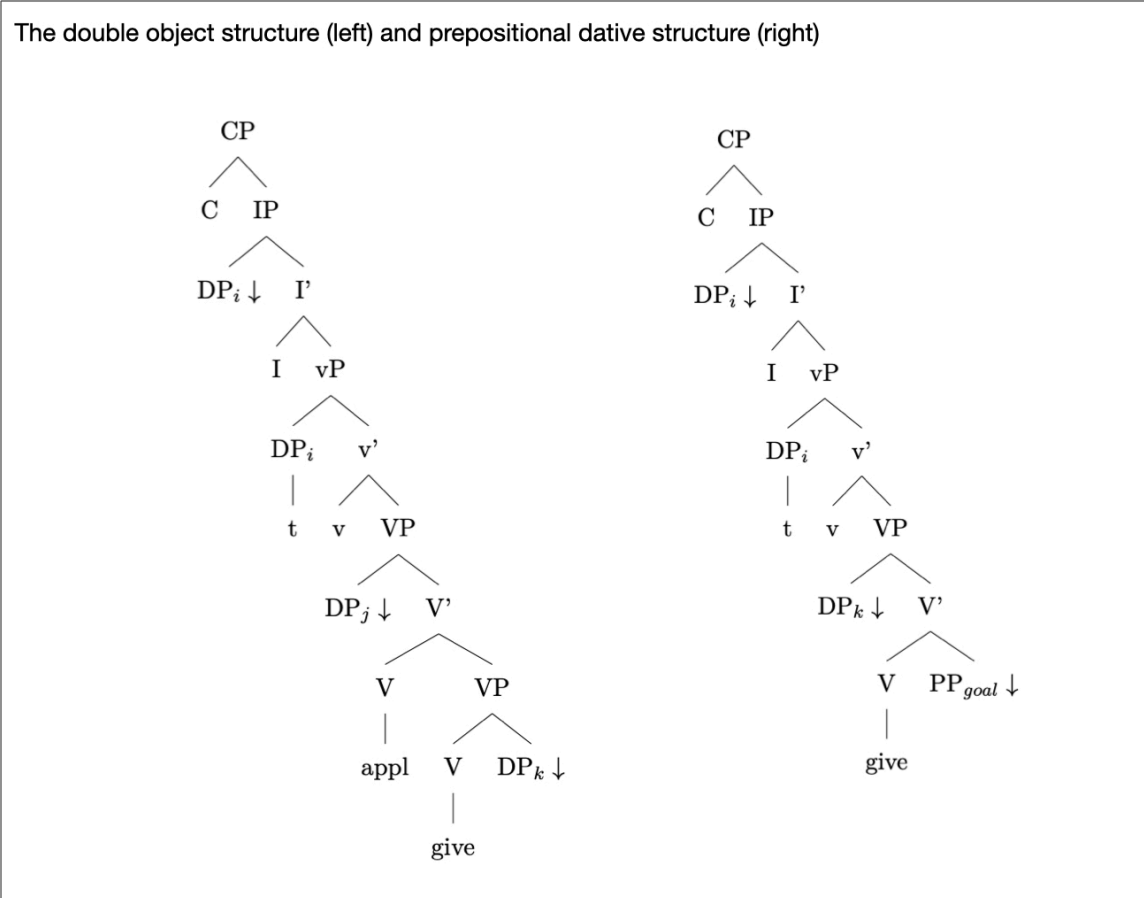

meant to be the abstract goal role, which includes finer-grained roles that can be described as recipient, beneficiary, and locational goal roles (Jackendoff (1985); Gruber (1965), see Levin \& Rappaport Hovav (2005)). The model included two verbs to be able to simulate the lexical boost effect, but only those two verbs to keep the model as simple as possible. Those conceptual nodes are connected to subtree level nodes. Subtree level nodes in the dative priming model include the nodes representing the applicative head, $P P_{\text {goal }}$, the lemmas of give and show, among other nodes that are not relevant (and hence omitted) here. The concept level node representing the goal role is connected to the subtree level nodes representing the applicative head and $P P_{\text {goal }}$ via excitatory connections. The concept level node representing the conceptual features of give and show are connected to the subtree level nodes representing the lemmas of give and 
show, respectively. Mutually exclusive nodes (give-show and appl- $P P_{\text {goal }}$ ) at the subtree level are connected via inhibitory links. Those subtree level nodes are connected to the elementary tree nodes, where elementary trees are represented (see Figure 8). Note that these dative structures involve the functional head known as little $v$, which is often assumed to introduce external arguments (Kratzer (1996); Chomsky (1995)) and is useful for accommodating three arguments in dative structures. For trees shown in other figures, little $v$ was omitted for simplicity.

In the dative priming model, lexically independent priming was simulated by increasing the weight of the connection between the GOAL node and either appl node or the $P P_{\text {goal }}$ node at the beginning of a simulation. If the connection between GOAL and appl is strengthened, the chance of selecting the DO structure increases (resulting in the DO priming). If the connection between $G O A L$ and the $P P_{\text {goal }}$ is strengthened, the chance of selecting the PD structure increases (resulting in the PD priming). Because those priming effects arise from the change in connection weights, they can be thought of as a form of implicit learning (Chang et al., 2006), and they are thus expected to be long-lasting (Bock \& Griffin (2000); Bock et al. (2007); Hartsuiker et al. (2008)) so long as processing other sentences intervening between prime and target sentences does not affect relevant connection weights.

The lexical boost effect for dative priming arises from residual activation of an elementary tree node used in parsing or producing a prime sentence. Residual activation was simulated by increasing the activation level of a relevant elementary tree at the beginning of a simulation. Because the elementary trees encoding the DO and PD structures are headed by verbs, residual activation affects the chance of DO or PD selection only when target sentences contain the same verb as prime sentences. Because the activation decays quickly, the lexical boost effect is expected to be short-lived, consistent with previous findings that suggest that the lexical boost effect disappears after a few intervening trials (Hartsuiker et al. (2008)).

The that-priming model was designed as follows. Conceptual nodes encode 
conceptual features associated with the presence of a complement clause (represented by the $\mathrm{X}$ node), conceptual features associated with the verbs think and say (represented by the THINK and SAY nodes, respectively), and conceptual features associated with the absence of wh-phrases in the embedded clause, or, alternatively, the presence of a truth-value-bearing feature of an embedded clause, which is absence when an embedded clause contains a wh-phrase (represented by the Y node). Just like in the dative priming model, those conceptual nodes are connected to subtree level nodes. subtree level nodes include the nodes representing that, the null complementizer (represented as null), the lemmas of think and say, among other nodes that are not relevant (and hence omitted) here. The $\mathrm{X}$ node is connected to the subtree level nodes representing that and the null complementizer (labeled as null), via excitatory connections. The THINK and SAY nodes are connected to the subtree nodes representing the lemmas of think and say, respectively. Those subtree level nodes are then connected to the elementary tree level nodes; T1 and T3 are connected to think, $\mathrm{T} 2$ and $\mathrm{T} 4$ are connected to say, T1 and T2 are connected to that and T3 and $\mathrm{T} 4$ are connected to $\mathrm{null}$, by virtue of those elementary trees containing subtree level representations as its elements. (see Figures 3 and 4).

In addition, the $\mathrm{Y}$ node is connected directly to the elementary tree nodes involved in sentences with cross-clausal filler-gap dependencies (T5, T6, A1-A4) via inhibitory connections. The idea here is that those elementary trees should not be selected unless target sentences contain cross-clausal filler-gap dependencies. Those elementary trees are, just like other elementary trees, connected to appropriate subtree level nodes. The decision to connect the Y-node directly to the elementary tree nodes was to minimize the number of nodes and connections in the model. There are other ways to implement the model without affecting its predictions, and I do not have theoretical commitment to this particular implementational decision. For example, the model can have two conceptual nodes, one boosting the activation of elementary trees for sentences with crossclausal filler-gap dependencies (T5-T6 and A1-A4) and the other boosting the activation of elementary trees for sentences without cross-clausal filler-gap de- 
pendencies (T1-T4). The connections between the Y-node and elementary tree nodes can also be mediated by subtree level nodes, if connection weights are configured properly.

In the that-priming model, the lexically independent priming effect arises from the strengthening of the connection between the $\mathrm{X}$ node and the that node or the null complementizer node. If the connection between the $\mathrm{X}$ node and the that node is strengthened, the chance of selecting the that complementizer increases (resulting in the that-priming effect). If the connection between the $\mathrm{X}$ node and the null complementizer node is strengthened, the chance of selecting the null complementizer increases (resulting in the null complementizer priming effect). The lexically dependent component of the that-priming arises from residual activation of the target elementary tree node used in parsing or producing prime sentences. Residual activation was simulated by increasing the activation level of the relevant elementary tree at the beginning of a simulation. Because elementary trees encoding the features of that or the null complementizer are headed by clause-taking verbs, residual activation affects the chance of selecting that or the null complementizer only when the target structure contains the same verb as the prime structure. Critically in TAG, elementary trees headed by the same verb are distinct, depending on whether their complement clause contains a gap. Thus, the model predicts that the lexical boost effect should be selective to when both prime and target sentences contain cross-clausal filler-gap dependencies and when neither does.

Simulation results of the dative priming model and the that-priming model (based on 100 simulated subjects, each with 20 trials per condition) are shown in Figure 9 and 10, respectively. The models were more likely to select the primed structures even when relevant verbs were not repeated between prime and target sentences, but priming effects were greater when relevant verbs are repeated. Thus, the current models capture the lexically independent priming and the lexical boost effect for dative priming and that-priming. Furthermore, the thatpriming model predicts that the lexical boost effect should disappear when either prime or the target sentences contain filler-gap dependencies crossing a clause 
boundary. This is because TAG derives bi-clausal sentences with and without cross-clausal filler-gap dependencies using two distinct elementary trees headed by clause-taking verbs. The current experiments were all designed to test this prediction.

Figure 9: The simulated dative priming effects.

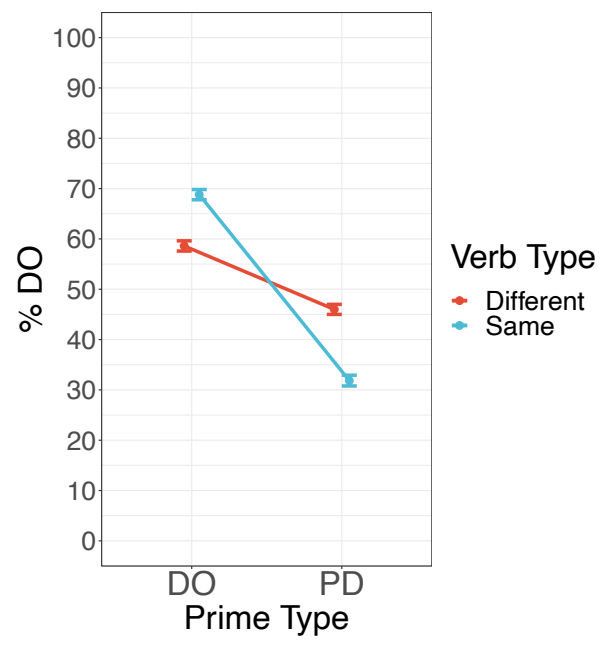

One potentially uncommon property of the current models is that they capture lexically independent structural priming using subtree level nodes representing parts of elementary trees. What are the rationales for this choice? There are at least two alternative approaches. The first alternative is to use combinatorial nodes (Pickering \& Branigan, 1998), which encode phrase-structure rules, instead of subtree level nodes. The $a p p l$ node and the $P P_{\text {goal }}$ node in the dative priming model can be replaced with the NP-NP combinatorial node and the NP-PP combinatorial node described in Pickering \& Branigan (1998), respectively. Also, the that node and the null complementizer nodes in the thatpriming model can be replaced with a combinatorial node encoding a phrase structure rule like $C P->C_{\text {that }} I P$ and a combinatorial node encoding a rule like $C P->C_{\text {null }} I P$. These replacements would not affect the predictions of the model. However, under the current TAG-based approach, it is conceptu- 
Figure 10: The simulated that-priming effects across different sentence types for prime and target sentences. The title of each graph represents the type of sentences used as prime and target sentences (in this order). noFG means sentences did not contain cross-clausal fillergap dependencies, and $F G$ means sentences did. For example, noFG-FG means the prime sentences were declarative sentences with no cross-clausal filler-gap dependency, and the target sentences contained a cross-clausal filler-gap dependency.
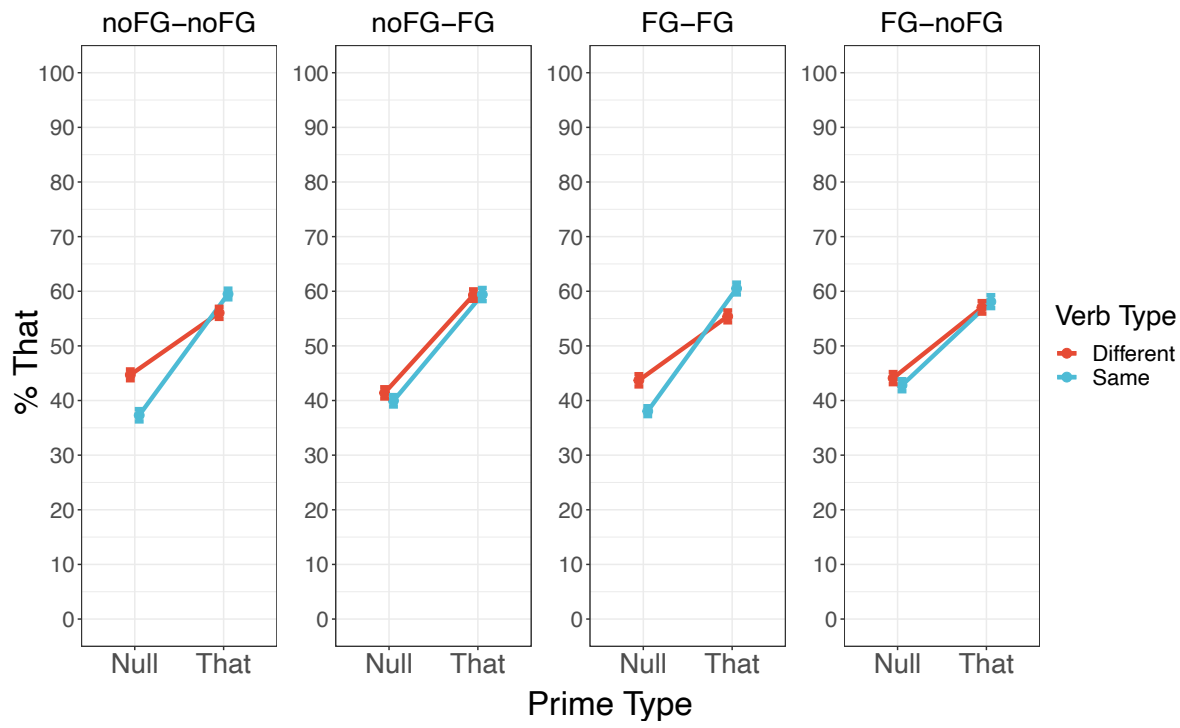

ally not natural to assume phrase structure rules and TAG elementary trees simultaneously in a single model, because phrase structure grammar and TreeAdjoining grammar are two distinct grammatical systems with different levels of expressive power. Also, in the current approach, the subtree level nodes and elementary tree level nodes have compositional relationships. If subtree level nodes encoding functional and phrasal categories are replaced with combinatorial nodes, this property would be lost. Thus, although subtree level nodes can be replaced with combinatorial nodes without affecting the model predictions, the choice to use subtree level nodes is conceptually more natural under the TAG framework.

The second alternative approach is to postulate nodes representing a set of elementary trees that are identical except for being headed by different words. If a set of elementary trees can be primed by a member of that set, the lexically 
independent structural priming may be captured without subtree level nodes. In fact, for dative priming, The $P P_{\text {goal }}$ node can be replaced with a node encoding a set of PD elementary trees, and the $a p p l$ node can be replaced with a node encoding a set of DO elementary trees. These replacements do not affect the prediction of the dative priming model. However, this approach makes a divergent prediction about the that-priming effect. Auxiliary trees headed by a clause-taking verb (like A1-A4) and non-auxiliary trees headed by the same clause-taking verb (like T1-T4 in Figure 7) do not constitute a single set of elementary trees that are identical except for being headed by different words. Thus, if the lexically independent priming effects are assumed to be due to the increased activation of a set of elementary trees that are identical except being headed by different words, the lexically independent that-priming effect is expected to be absent when only prime or target sentences contain cross-clausal filler-gap dependencies. To the extent that this prediction is incorrect, the current approach that assumes subtree level nodes is empirically better than the alternative approach that assumes the priming of sets of elementary trees. As shown below, the lexically independent that-priming occurs even when only a prime or target sentence contains a cross-clausal filler-gap dependency. Thus, I will not discuss the alternative approach that assumes nodes representing a set of elementary trees any further in the current paper.

Finally, the current model architecture can be used for modeling other types of structural priming effects. For example, the lexically independent activepassive priming effect (Bock, 1986) can be modeled as the strengthening of the connection between a concept level node encoding agentivity and a subtree level node encoding a functional head for the active voice or the passive voice, or alternatively, a preposition by. The lexical boost effect for active-passive priming (e.g., Segaert et al. (2011); Messenger et al. (2012)) can be captured by residual activation of an elementary tree for active or passive sentences. Similarly, the lexically independent priming of genitive structures (e.g., the girl's apple vs. the apple of the girl, Bernolet et al. $(2012,2013))$ can be due to the strengthening of the connection between a concept level node encoding possession and a subtree 
level node encoding 's or of. The lexical boost effect for genitive structural priming can also be captured if an elementary tree for the girl's apple and the apple of the girl involve distinct elementary trees headed by the head noun girl. Finally, the lexically independent priming of locative alternations (e.g., the girl loaded the truck with hay vs. the girl loaded hay onto the track, Chang et al. (2003)) can be due to the strengthening of the concept level node encoding the goal role and a subtree level node encoding an applicative head or a locative preposition. The lexical boost effect for locative priming can also be captured because two structures are usually assumed to involve distinct elementary trees. Thus, the current approach has the potential to capture a diverse set of lexically independent and lexically dependent structural priming effects (but see General Discussion for potential limitations of the current approach).

\section{Current study: an overview}

The experiments reported here share the same task, and they together test the critical prediction of the TAG-based model introduced above. Here I describe the experimental task used in those experiments and the goal of each experiment to give an overview of the current study.

In all experiments, a variant of the sentence recall task, similar to the one used by Ferreira (2003) was used. In this task, participants first read aloud two bi-clausal sentences, presented one at a time for five seconds, and then they were presented with memory cues that are uniquely associated with one of the two sentences as a prompt to recall one of the memorized sentences (Figure 11). In critical trials, sentences that were presented first were always target sentences to be recalled, and sentences that were presented second were always prime sentences. This ensures that prime sentences immediately preceded target production. Filler trials ensured that speakers were asked to recall the first and the second sentences equally often. Recall responses (that met certain criteria described below) were coded for whether they included the complementizer that or the null complementizer. Experiment 1 tested if the lexical boost effect for 
that-priming can be observed at all when both prime and target sentences did not contain cross-clausal filler-gap dependencies. Experiment 2 tested if the lexical boost effect in Experiment 1 disappears when only prime sentences contain cross-clausal filler-gap dependencies. Experiment 3 tested if the lexical boost effect reappears when both target and prime sentences contain cross-clausal fillergap dependencies. Experiment 4 tested if the lexical boost effect in Experiment 3 disappears when prime sentences are wh-questions but without cross-clausal filler-gap dependencies. Experiment 5 tested if the lexical boost effect still fails to occur when only prime sentences contained cross-clausal filler-gap dependencies, despite clause-taking verbs in prime and target sentences sharing the same inflectional form. Those experiments together test the prediction of the TAGbased model that the lexical boost effect should be selectively observed when both prime and target sentences contain cross-clausal filler-gap dependencies or when neither does.

\section{Data availability}

Data, scripts, and materials used in this study are available here:

https://osf.io/us4wg/?view only=5a11ac1033c94473a988263427b31119

\section{Experiment 1}

Experiment 1 tested if the lexical boost effect occurs when a clause-taking verb is repeated between prime and target sentences. In Experiment 1, both prime and target sentences were sentences with no cross-clausal filler-gap dependencies, like (1a) and (1b).

(1) a. The manager announced/implied (that) he would promote the employee. [Prime]

b. The director announced (that) the actor would be in the new movie. [Target] 
Figure 11: A schematic illustration of the task. Sentences and recall cues are examples from the null-same condition in the critical trials in Experiment 1.

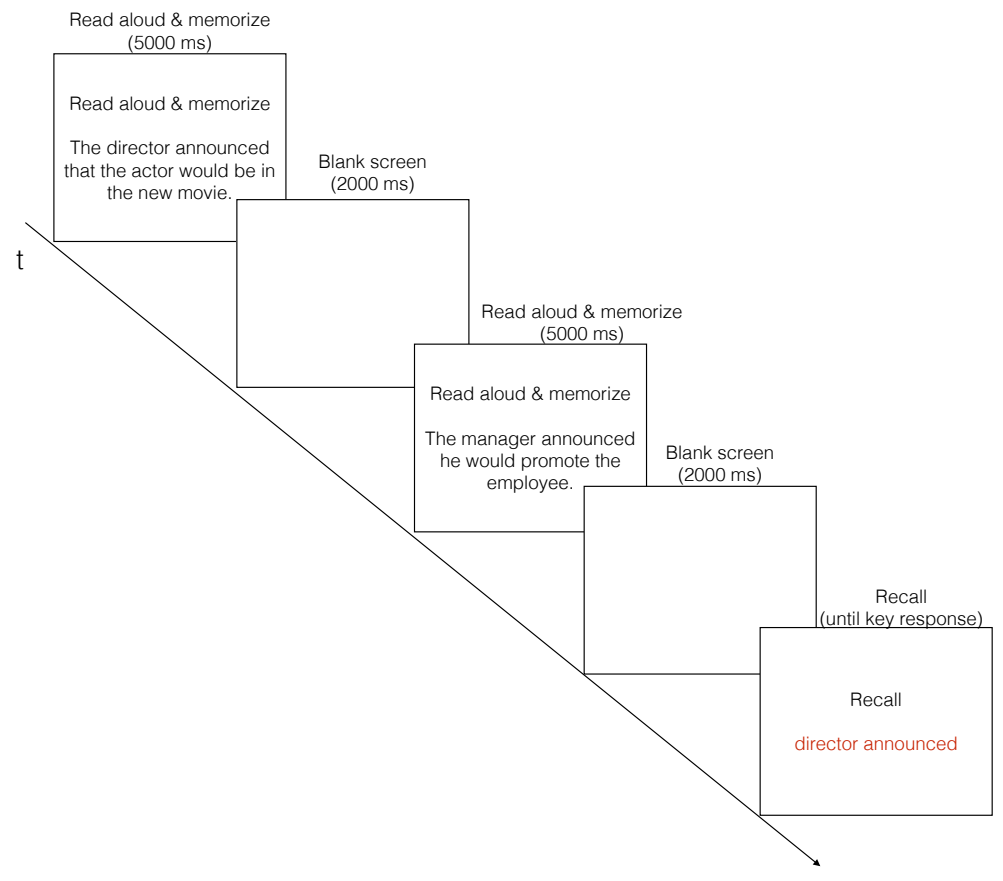

If the lexical boost effect is observed, Experiment 1 provides the point of comparison for subsequent experiments that tested if the lexical boost effect disappears when only either prime or target sentences contain cross-clausal fillergap dependencies.

\subsection{Method}

\subsubsection{Participants}

Eighty monolingual English speakers were recruited using Prolific Academic.

${ }^{3}$ For each participant, informed consent was obtained. Each experiment took

\footnotetext{
${ }^{3}$ The effect size for the critical effects was not known a priori, so I arbitrarily decided to recruit sixty participants for Experiments 1 and 2, which were run partly in parallel. But due to a list imbalance in Experiment 1, the number of participants was increased to eighty. In Experiments 3 and 4, eighty participants were recruited simply to match the number of participants in Experiment 1. In Experiment 5, given the increased complexity of the target
} 
roughly 30 minutes. Each participant was paid $\$ 5$ for compensation. Two participants were replaced due to their failure to follow instructions or because they produced one or no scorable response in one or more conditions ( $i 33 \%$ ).

\subsubsection{Materials}

In Experiment 1, twenty-four pairs of bi-clausal sentences like (1a) and (1b) were constructed. Both prime and target sentences were bi-clausal declarative sentences with definite noun phrases filling appropriate argument slots. Across all experimental items, there were twelve unique clause-taking verbs: announce, argue, assume, believe, claim, fear, guess, hear, imply, say, suspect, think, each used twice in critical trials. The verbs of embedded clauses in the prime sentences (e.g., promote in $1 \mathrm{~b}$ ) were always transitive because, in subsequent experiments, those sentences were recycled to create minimally different sentences with cross-clausal filler-gap dependencies (e.g., Who did the manager announce he would promote?). The matrix verbs of target sentences were same (e.g., announced in 1b) or different (e.g., implied in 1b) from the matrix verbs of the paired prime sentences. The prime sentence contained that or not. See Appendix B for the full list of stimuli. Half of the target sentences contained that, and the other half did not, so the probability of seeing that in target sentences was exactly $50 \%$. When creating recall cues, the head noun of the matrix subject and the matrix verb were used (e.g., director announced for the example sentence $(1 b))$.

Thirty-six additional sentence pairs were created as filler items. Twenty-four of those fillers had bi-clausal structures and were virtually indistinguishable from the critical items from the participants' perspective. For those filler sentence pairs, just like in critical items, recall cues corresponded to the subject noun head and the matrix verb. However, recall cues always corresponded to the second sentence (which would correspond to the prime sentence in the experimental trials) for those filler items. This is to ensure that participants cannot predict

sentences, I anticipated a higher rate of non-scorable responses and participant exclusion, so I recruited ten participants more than in Experiments 1, 3, and 4. 
which sentence (the first or the second) they need to recall before seeing a memory cue. Twelve of the filler items were all mono-clausal dative sentences, like The landlord loaned the new tenant an old sofa. Those were added to increase the diversity of sentences participants were asked to recall.

\subsubsection{Procedure}

The experiment was run online using Penn Controller Ibex (PCIBex; Zehr \& Schwarz (2018)). Participants were first given the following instruction:

In each trial in this experiment, you will see two sentences, one at a time. Your task is to read aloud and memorize the two sentences for later recall. You are given 5 seconds to read aloud and memorize each sentence. After you read aloud and memorize two sentences, you will be prompted to recall one of them. The prompt will be 2-3 words that appeared in one of the two sentences. For example, if you memorized 'The boy went to the store' and 'The dog chased the cat', you may see 'boy went' as the prompt to recall the sentence 'the boy went to the store.' Please recite the sentence that included the prompt words aloud.

Following this instruction, participants went through three practice trials, which had the same task structure as the experimental trials. Following the practice trials, the main experimental trials began, which had the following sequence of events. First, a sentence (a target sentence in critical trials) was presented for 5 seconds. After 2 seconds of a blank screen, another sentence (a prime sentence in critical trials) was presented for 5 seconds. After another 2 seconds of a blank screen, the recall cue (e.g., director announced) was presented. Participants were given as much time as they needed to recall the sentence, and the recall cue was kept on the screen until they pressed the space key. Participants' voice responses were recorded using participants' microphones. After a trial was completed, participants pressed the "Next" button to move on to the next trial. The task structure is illustrated in Figure 11. 


\subsection{Scoring and analyses}

All audio files were first transcribed and coded for scorability and complementizer type. Responses were considered scorable as long as they contain bi-clausal structures with the appropriate embedding verb (which was given as part of the recall cues). Even when the responses were incomplete or did not faithfully reproduce the target sentences, they were considered scorable as long as there was clear evidence that speakers attempted to say a bi-clausal structure and said a portion of the embedded clause (e.g., when only the embedded subject was spoken). Responses were considered non-scorable when they produced a structure that did not allow the use of that (e.g., the director announced who would be in the new movie was considered non-scorable). Unintelligible responses and no responses were also considered non-scorable. When participants recited sentences more than once, only the first scorable response was counted. Scorable responses were then coded for complementizer type by the author who was blind to which prime conditions each response belonged to. Responses with the complementizer that were coded as 1 , and responses with the null complementizer were coded as 0 .

Scorable responses were submitted to statistical analysis. All analyses were conducted in R (R Core Team (2020)), using the brms package (Bürkner, 2018). A Bayesian hierarchical model assuming the Bernoulli distribution was constructed with complementizer choice as the variable of interest, and Prime Type (null vs. that) and Verb Type (Different vs. Same) as critical fixed effects. In addition, whether the original target sentence contained that or the null complementizer (Target Type) and its interaction with other factors were included as fixed effects, but by-subject or by-item random slopes for effects involving Target Type were not included in the model, because Target Type was not our primary theoretical interest and it was included merely as a control predictor. By-subject and by-item random intercepts and random slopes for the Prime Type, Verb Type, and their interactions were included in the random effects structure (cf. Barr et al. (2013)). For the overall analysis, Prime Type (null vs. that), Verb Type (Different vs. Same), and Target Type (null vs. that) were 
coded as -0.5 and 0.5 .

For all analyses, we used the $95 \%$ credible interval (based on percentiles) to make inferences. That is, we conclude that there is evidence for an effect if the $95 \%$ credible interval for that effect does not include 0. For each model, four Monte Carlo Markov Chains with 5000 samples were run. The first 2500 samples were discarded as a warm-up period. All priors were normal distributions with a mean value of 0 . The variance on the prior distribution was set to 2 for all fixed effects, and 10 for the intercepts. The prior on the correlation matrix was a LKJ regularizing prior (Lewandowski et al. (2009)) with $\eta=2$ (Vasishth et al. (2018)). For all models reported below, the $\hat{R}$ statistic was at or near 1.0 for all fixed effects parameters of interest, and no divergences were observed.

\subsection{Results}

In Experiment 1, $4.2 \%$ of the trials (81 out of 1920 trials) were identified as non-scorable, so they were excluded from subsequent analyses. The rates of non-scorable responses across four conditions were as follows: Null-Different: 4.6\%; Null-Same: 4.0\%; That-Different: 4.6\%; That-Same: $3.8 \%$.

The rate of that production across conditions is summarized in Figure 12. Table 1 reports the statistical results. In the different verb condition, speakers on average produced that $44.8 \%$ of the time given null complementizer primes. In contrast, speakers on average produced that $58.4 \%$ of the time given that primes. That is, there was a $13.6 \%$ structural priming effect when there was no overlap in the embedding verb between prime and target sentences. In the same verb condition, speakers produced that $44.2 \%$ of the time given null complementizer primes. In contrast, speakers produced that $67.2 \%$ of the time given that primes. That is, there was a $23.0 \%$ structural priming effect when the matrix verb was repeated. This means that the structural priming effect was overall $9.4 \%$ bigger when the matrix verbs were repeated than when they were not repeated. Reflecting this pattern, there was evidence for the two-way interaction between Prime Type and Verb Type, and this two-way interaction did not interact with Target Type (i.e., there was no three-way interaction). There was also evidence 
for the main effect of Prime Type. There was some evidence for the main effect of Verb Type. However, I avoid interpreting those main effects due to the interaction involving those terms. Also, there was evidence for the main effect of Target Type, simply suggesting that speakers had some memory of whether the sentences they saw contained that or not. There was no evidence for other effects.

To test if the current data provide evidence for the lexically independent priming, a nested model evaluated the effect of Prime Type within each level of Verb Type. This analysis revealed evidence for the effect of Prime Type in the different verb condition $(\hat{\beta}=0.84,95 \% \mathrm{CrI}=[0.49,1.19], \operatorname{Pr}(\beta>0)>0.99)$ and in the same verb condition $(\hat{\beta}=1.43,95 \% \mathrm{CrI}=[1.07,1.80], \operatorname{Pr}(\beta>$ $0)>0.99$ ), suggesting that, although the magnitude of the priming effect was larger when the embedding verb was repeated, the complementizer choice can be primed without repeating verbs.

Table 1: The statistical results in Experiment 1. 95\% credible intervals that did not include zero are bold-faced. The R-style model formula was: that $\sim$ PrimeType*VerbRepetition*TargetType $+(1+$ PrimeType*VerbRepetition $\mid$ Subject $)+(1+$ PrimeType*VerbRepetition | Item)

\begin{tabular}{llll}
\hline Term & Estimate & Est. Error & $95 \%$ CrI \\
\hline Intercept & 0.17 & 0.22 & {$[-0.26,0.61]$} \\
Prime Type & 1.15 & 0.14 & {$[\mathbf{0 . 8 7}, \mathbf{1 . 4 3}]$} \\
Verb Type & 0.25 & 0.14 & {$[-0.01,0.52]$} \\
Target Type & 1.77 & 0.33 & {$[\mathbf{1 . 1 2}, \mathbf{2 . 4 2}]$} \\
Prime Type x Verb Type & 0.60 & 0.26 & {$[\mathbf{0 . 0 9} \mathbf{1 . 1 2}]$} \\
Prime Type x Target Type & -0.46 & 0.27 & {$[-0.99,0.06]$} \\
Verb Type x Target Type & 0.10 & 0.27 & {$[-0.42,0.62]$} \\
Prime Type x Verb Type x Target Type & -0.26 & 0.52 & {$[-1.28,0.75]$} \\
\hline
\end{tabular}

\subsection{Discussion}

The results of Experiment 1 showed that the choice of complementizer can be primed both with and without repeating a clause-taking verb between target and prime sentences and that this priming effect can be lexically boosted. This 
Figure 12: The by-subject mean percentage that production across conditions in Experiment 1. The left panel shows the data from trials where target sentences contained no that. The middle panel shows the data from trials where target sentences contained that. The right panel shows the data from all trials. The error bars represent the by-subject standard error of the means.

\section{Experiment 1 (noFG-noFG)}

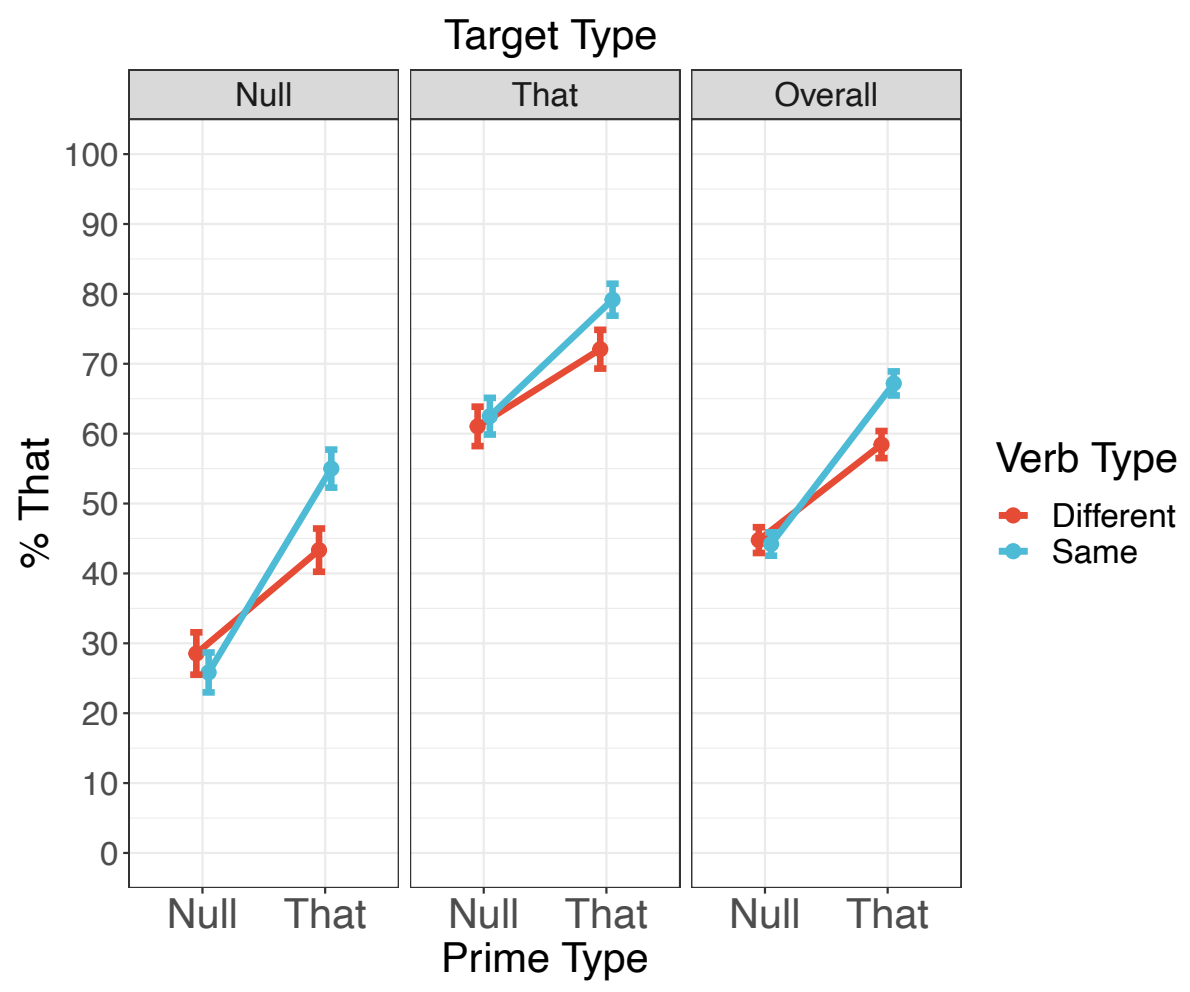

replicates and extends Ferreira (2003). As discussed above, the TAG-based model predicts that the lexical boost effect for that-priming should disappear when the embedded clauses of either prime or target sentences contain crossclausal filler-gap dependencies. Thus, Experiment 1 established that the lexical boost effect occurred when there was no filler-gap dependency in prime or target sentences and provided the point of comparison for subsequent experiments.

To the extent that this lexical boost effect is specific to the repetition of clause-taking verbs that select the complementizer type (contrary to Scheep- 
ers et al. (2017) and Chang et al. (2006)), the current results suggest that clause-taking verbs like think encodes the distinction between that clauses and that-less clauses. In the current experiment, the lexical boost effect primarily appeared as the increase in the that production rather than the increase in the null complementizer production. This is somewhat surprising given Ferreira (2003). Ferreira (2003) found that the lexically independent complementizer priming was bi-directional, that is, that primed that and the null complementizer primed the null complementizer, compared to a neutral baseline. Indeed, Ferreira (2003) found that the magnitude of the priming effect was larger for null complementizer priming than that-priming. In the current experiment, it cannot be known whether the lexically independent priming effect was caused by the priming of that, the null complementizer, or both because there was no neutral baseline. In that sense, the current results are not necessarily inconsistent with the pattern reported by Ferreira (2003). However, given that the null complementizer can be primed, the question is why the lexical boost effect did not enhance the null complementizer priming effect. One possibility is that the null complementizer priming was stronger than the that-priming at the baseline. It may be difficult to make the null complementizer priming even stronger by repeating a clause-taking verb.

A more concerning possibility is that the lexical boost effect was due to the priming of bigrams like thought that. This possibility is naturally compatible with the result that the lexical boost effect enhanced the priming of that, but not the priming of the null complementizer. If this potential bigram priming is sufficient to explain the lexical boost effect reported here, the current results do not necessarily license claims about lexical or syntactic representations. This issue will be addressed in subsequent experiments.

\section{Experiment 2}

Experiment 1 established that complementizer choice can be primed without repeating clause-taking verbs, but the priming effect can be boosted by 
repeating them. Experiment 2 tested the critical prediction of the TAG-based model that the lexical boost effect should disappear when either prime or target sentences (but not both) contain a cross-clausal filler-gap dependency. This prediction derives from the claim of TAG theories that elementary trees headed by verbs like think used for adjoining (for sentences with cross-clausal filler-gap dependencies) and substitution (for sentences without cross-clausal filler-gap dependencies) are distinct. To test this prediction, in Experiment 2, we changed the prime sentences from Experiment 1 so that they contain a cross-clausal filler-gap dependency, as in the following example.

(2) Who did the manager announce/imply (that) he would promote?

Thus, in Experiment 2, prime sentences always contained cross-clausal fillergap dependencies crossing the complementizer structure, as who is the object of the verb in the embedded clause (promote). Target sentences were the same as in Experiment 1 and did not contain any filler-gap dependency.

\subsection{Method}

\subsubsection{Participants}

Sixty monolingual English speakers were recruited using Prolific Academic.

\subsubsection{Materials}

Materials from Experiment 1 were reused with minimal modifications. In Experiment 2, the prime sentences of Experiment 1 were converted to sentences with object-extracted wh-questions, like Who did the manager announce/imply (that) he would promote?, by replacing embedded object noun phrases with who, moving it to the front of the sentence, adding did after who and the question mark at the end. Twenty-four of the thirty-six filler sentences were modified so they looked indistinguishable from new target sentences; the first sentence of each sentence pair was always a wh-question and the second sentence was always a declarative sentence. 


\subsubsection{Procedure, scoring and analyses}

The experimental and analysis procedures were the same as in Experiment 1. The scoring criteria were also the same.

\subsection{Results}

In Experiment 2, $5.8 \%$ of the trials (83 out of 1440 trials) were identified as non-scorable, so they were excluded from subsequent analyses. The rates of non-scorable responses across four conditions were as follows: Null-Different: 5.3\%; Null-Same: 5.5\%; That-Different: 6.7\%; That-Same: 5.5\%.

The rates of that production across conditions is summarized in in Figure 13. Table 2 reports the statistical results. In the different verb condition, speakers on average produced that $52.4 \%$ of the time given null complementizer primes. In contrast, speakers on average produced that $72.8 \%$ of the time given that primes. That is, there was a $20.4 \%$ structural priming effect when clause-taking verbs were not repeated. In the same verb condition, speakers produced that $52.2 \%$ of the time given null complementizer primes. In contrast, speakers produced that $69.7 \%$ of the time given that primes. That is, there was a $17.5 \%$ structural priming effect when the matrix verb was repeated. This means that the structural priming effect was overall $2.9 \%$ smaller when the matrix verbs were repeated than when they were not repeated. There was evidence for the main effect of Prime Type without any interaction involving this term, suggesting that there was overall priming of that regardless of whether the prime verb was repeated or not and regardless of whether the original sentence contained that or not. There was also evidence for the main effect of Target Type, simply suggesting that speakers had some memory of whether the memorized sentences contained that or not. There was no evidence for other effects.

A nested model evaluated the effect of Prime Type within each level of Verb Type. This analysis revealed evidence for the effect of Prime Type in the different verb condition $(\hat{\beta}=1.50,95 \% \mathrm{CrI}=[1.00,2.04], \operatorname{Pr}(\beta>0)>0.99)$ and in the same verb condition $(\hat{\beta}=1.15,95 \% \mathrm{CrI}=[0.70,1.62], \operatorname{Pr}(\beta>0)>$ 0.99). Thus, verb repetition is not necessary for the that-priming effect to occur. 
Finally, a model directly compared the magnitude of the lexical boost effect in Experiment 1 and Experiment 2. The fixed effects of this model included Prime Type, Verb Type, Target Type, Experiment, and their interactions. The random-effects structure included by-subject and by-item random intercepts, as well as the by-subject random slopes for Prime Type, Verb Type, and their interactions, and the by-item random slope of Prime Type, Verb Type, Experiment, and their interactions. The factor Experiment was coded as -0.5 (Experiment 1) and 0.5 (Experiment 2). The results of this analysis are summarized in Table 3. Most relevantly, there was evidence for the three-way interaction between Prime Type, Verb Type, and Experiment, suggesting that the magnitude of the lexical boost effect was larger in Experiment 1 than in Experiment 2. Aside from the critical three-way interaction, there was evidence for the main effects of Prime Type, Target Type, and Experiment. I avoid interpreting these main effects because there was evidence for interactions involving each of those factors. There was also evidence for the two-way interaction between Target Type and Experiment, but again, given the evidence for the higher-order interaction, I avoid giving an interpretation to this effect. Finally, there was evidence for the two-way interaction between Prime Type and Target Type, suggesting that the

priming effect was bigger when the memorized target sentence did not contain that.

\subsection{Discussion}

Experiment 2 showed that the lexical boost effect disappeared when prime sentences contained a cross-clausal filler-gap dependency. This confirms the prediction of the TAG-based model, suggesting that there are two distinct structures headed by verbs like think depending on whether sentences contain crossclausal filler-gap dependencies.

The explicit memory account of the lexical boost effect struggles to capture the contrast between Experiments 1 and 2. The explicit memory account predicts that the lexical boost effect should be observed both in Experiments 1 and 2 because verbs like think were repeated in the same verb condition in 
Table 2: The statistical results in Experiment 2. 95\% credible intervals that did not include zero are bold-faced. The R-style model formula was: that $\sim$ PrimeType*VerbRepetition*TargetType $+(1+$ PrimeType*VerbRepetition $\mid$ Subject $)+(1+$ PrimeType*VerbRepetition | Item)

\begin{tabular}{llll}
\hline Term & Estimate & Est. Error & $95 \%$ CrI \\
\hline Intercept & 0.81 & 0.26 & {$[\mathbf{0 . 3 1}, \mathbf{1 . 3 1}]$} \\
Prime Type & 1.38 & 0.21 & {$[\mathbf{1 . 0 0} \mathbf{1 . 8 1}]$} \\
Verb Type & -0.14 & 0.18 & {$[-0.50,0.22]$} \\
Target Type & 1.13 & 0.30 & {$[\mathbf{0 . 5 2}, \mathbf{1 . 7 2}]$} \\
Prime Type x Verb Type & -0.33 & 0.38 & {$[-1.09,0.42]$} \\
Prime Type x Target Type & -0.45 & 0.32 & {$[-1.08,0.18]$} \\
Verb Type x Target Type & 0.00 & 0.34 & {$[-0.65,0.65]$} \\
Prime Type x Verb Type x Target Type & 0.32 & 0.63 & {$[-0.92,1.56]$} \\
\hline
\end{tabular}

Table 3: The results of the joint analyses of Experiments 1 and 2. 95\% credible intervals that did not include zero are bold-faced. The R-style model formula was: that $\sim$ PrimeType*VerbRepetition*TargetType*Experiment + (1+PrimeType*VerbRepetition | Subject) $+(1+$ PrimeType*VerbRepetition*Experiment | Item $)$

\begin{tabular}{llll}
\hline Term & Estimate & Est. Error & $95 \%$ CrI \\
\hline Intercept & 0.48 & 0.20 & {$[\mathbf{0 . 0 9}, \mathbf{0 . 8 7}]$} \\
Prime Type & 1.24 & 0.12 & {$[\mathbf{1 . 0 1}, \mathbf{1 . 4 8}]$} \\
Verb Type & 0.08 & 0.11 & {$[-0.15,0.30]$} \\
Target Type & 1.43 & 0.30 & {$[\mathbf{0 . 8 5}, \mathbf{2 . 0 1}]$} \\
Experiment & 0.60 & 0.26 & {$[\mathbf{0 . 0 9}, \mathbf{1 . 1 2}]$} \\
Prime Type x Verb Type & 0.17 & 0.22 & {$[-0.26,0.59]$} \\
Prime Type x Target Type & -0.43 & 0.21 & {$[-\mathbf{0 . 8 5}, \mathbf{- 0 . 0 2}]$} \\
Verb Type x Target Type & 0.05 & 0.23 & {$[-0.39,0.50]$} \\
Prime Type x Experiment & 0.16 & 0.23 & {$[-0.29,0.61]$} \\
Verb Repetition x Experiment & -0.35 & 0.20 & {$[-0.75,0.05]$} \\
Target Type x Experiment & -0.72 & 0.22 & {$[\mathbf{- 1 . 1 5}, \mathbf{- 0 . 3 0}]$} \\
Prime Type x Verb Type x Target Type & 0.04 & 0.42 & {$[-0.79,0.90]$} \\
Prime Type x Verb Type x Experiment & -0.86 & 0.41 & {$[\mathbf{- 1 . 6 6 , - 0 . 0 6}]$} \\
Prime Type x Target Type x Experiment & -0.10 & 0.40 & {$[-0.90,0.70]$} \\
Verb Type x Target Type x Experiment & -0.05 & 0.39 & {$[-0.81,0.73]$} \\
Prime Type x Verb Type x Target Type x Experiment & 0.62 & 0.75 & {$[-0.85,2.11]$} \\
\hline
\end{tabular}


Figure 13: The by-subject mean percentage that production across conditions in Experiment 2. The left panel shows the data from trials where target sentences contained no that. The middle panel shows the data from trials where target sentences contained that. The right panel shows the data from all trials. The error bars represent the by-subject standard error of the means.

\section{Experiment 2 (FG-noFG)}

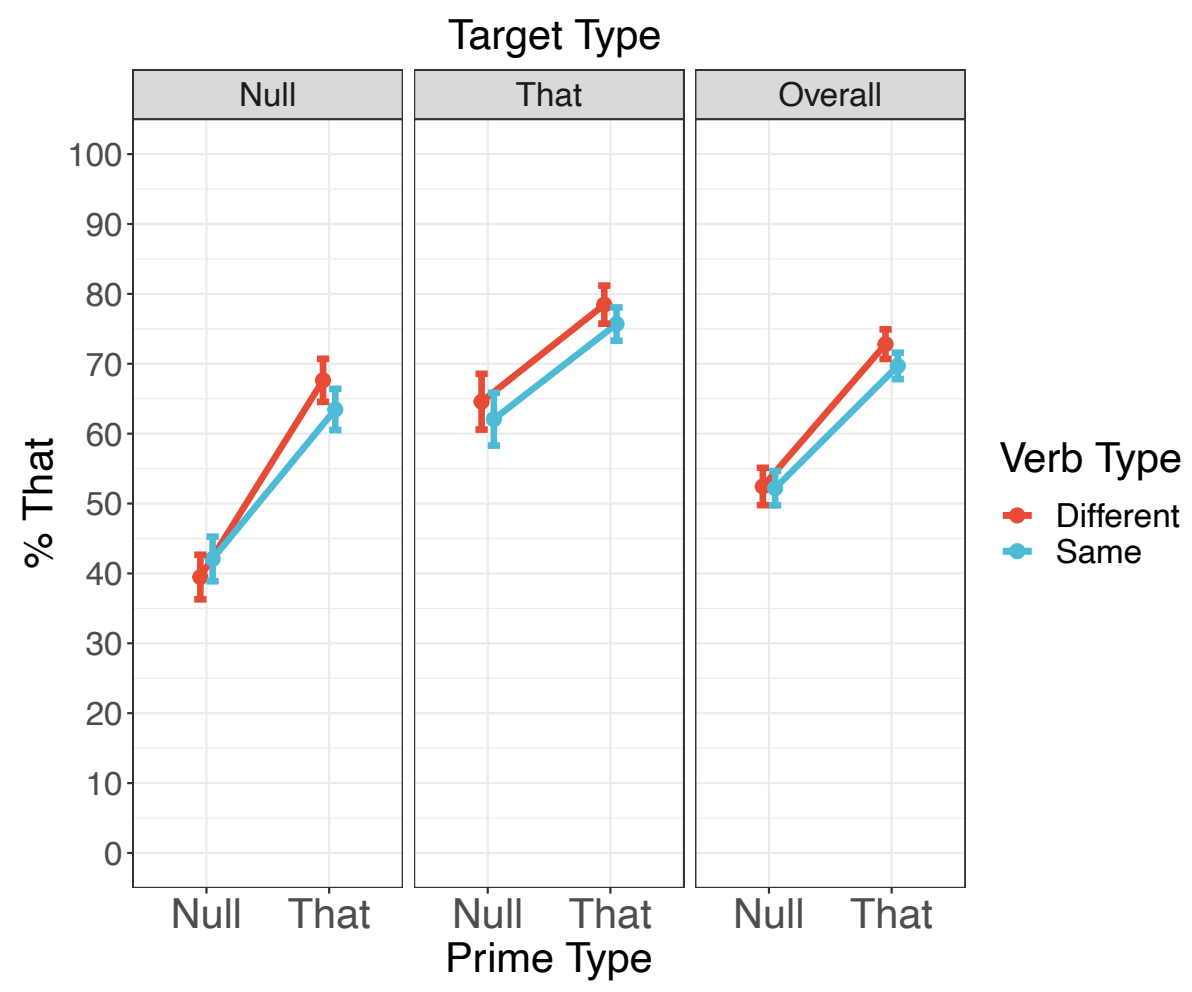

Experiments 1 and 2 alike. Contrary to this prediction, the lexical boost effect was selectively observed in Experiment 1.

The bigram account of the lexical boost effect still cannot be ruled out, although it needs an additional assumption to capture the contrast between Experiment 1 and Experiment 2. In particular, in the same verb condition in Experiment 2, strings like think that appeared in the prime sentences and strings like thought that appeared in the target sentences. Those strings are identical at the lemma level, but not at the form (lexeme) level. Thus, to 
maintain the bigram account of the lexical boost effect, it must be assumed that relevant bigrams must be identical at the form level to trigger the lexical boost effect-like pattern. This account is further evaluated in Experiments 4 and 5. However, it should be noted that Pickering \& Branigan (1998) showed that the inflectional form of the verb did not modulate the lexical boost effect in dative priming experiments. Thus, even the lexeme-level bigram account cannot be taken as the general explanation of all lexical boost effects across different priming studies.

Taken together, Experiment 2 confirmed the critical prediction of the TAGbased model that the lexical boost effect for complementizer priming should disappear when either prime or target sentences contain cross-clausal filler-gap dependencies. However, potential alternative explanations and potential confounds remain. The bigram account of the lexical boost effect was not conclusively ruled out because, in the same verb condition, prime and target sentences share lemma level bigrams. Additionally, although the explicit memory account of the lexical boost effect has difficulty accounting for the contrast between Experiments 1 and 2, the account may be salvaged by assuming that the explicit memory of prime sentences is quickly lost before the target production (and therefore becomes not potent for causing priming) when prime sentences are complex. Subsequent experiments address those issues.

Aside from the difference in the lexical boost effect, there were some notable differences between Experiments 1 and 2. In Experiment 2, speakers were more likely to use that than in Experiment 1 when target sentences contained no that. This is probably because of the increased task difficulty of Experiment 2 compared to Experiment 1. Experiment 2 was probably more difficult than Experiment 1, given that the prime sentences in Experiment 2 contained fillergap dependencies. Sentences with filler-gap dependencies likely involve complex comprehension processes (Fodor (1978); Frazier et al. (1983); Frazier \& Flores d'Arcais (1989); Garnsey et al. (1989); Aoshima et al. (2004); Omaki et al. (2015); Clifton \& Frazier (1989); Wanner \& Maratsos (1978)) and therefore they are likely harder to comprehend and memorize. With complex prime sentences, 
which intervened between the memorization and recall of target sentences, the accurate maintenance and retrieval of target sentences in memory are expected to be harder. This may especially affect the memory trace of the null complementizer in target sentences because the null complementizer is semantically and phonologically so impoverished that its memory trace may be easily lost or overwritten. Relatedly, both in Experiments 1 and 2, priming effects were larger when the original target sentences contained no that. This pattern makes sense if the null complementizer leaves only fragile memory traces that can be easily lost or overwritten.

\section{Experiment 3}

Experiments 1 and 2 together showed that the lexical boost effect for the that-priming effect occurred, but not when only prime sentences contained a cross-clausal filler-gap dependency. This pattern confirms the prediction of the TAG-based model. However, one concern was that prime sentences in Experiment 2 were more complex because they contained cross-clausal filler-gap dependencies. The increased complexity of prime sentences may have weakened or eliminated the lexical boost effect in Experiment 2 because the explicit memory of complex prime sentences could be hard to maintain. To address this concern, Experiment 3 investigated if the lexical boost effect occurs when both prime and target sentences contain cross-clausal filler-gap dependencies. In Experiment 3, prime sentences were identical to those in Experiment 2, but target sentences were modified to contain a cross-clausal filler-gap dependency, as in the following:

(3) Who did the manager announce/imply (that) he would promote? [Prime]

(4) Who did the director announce (that) he would nominate? [Target]

The TAG-based model predicts the lexical boost effect selectively when both prime and target sentences contained cross-clausal filler-gap dependencies. If the lexical boost effect is observed in Experiment 3, the concern that the increased 
complexity of prime sentences weakened the lexical boost effect can be ruled out, because prime sentences in Experiment 3 were identical to those in Experiment 2 .

6.1. Method

\subsubsection{Participants}

Eighty monolingual English speakers were recruited using Prolific Academic. Four participants were replaced due to their failure to follow the instruction or because they produced one or no scorable response in one or more conditions (i $33 \%)$.

\subsubsection{Material}

Target sentences in Experiment 3 were constructed by modifying target sentences from Experiments 1 and 2. To modify target sentences from Experiments 1 and 2 to embedded object wh-questions, some aspects of target sentences needed to be changed. For example, when the sentences in Experiments 1 and 2 did not contain the embedded transitive verbs, the embedded verbs had to be changed to transitive verbs. This change was accompanied by changes in the arguments of those verbs to keep the sentences mostly naturally sounding. See Appendix B for the full list of materials.

\subsubsection{Procedure, scoring and analyses}

The experimental and analysis procedures were the same as in Experiments 1 and 2. Prime sentences were identical to those in Experiment 2. Scoring criteria were also the same as in Experiments 1 and 2. However, in Experiment 3, because target sentences were more complex than in Experiments 1 and 2, there were a fair number of trials where participants produced structures that do not permit the complementizer that (e.g., sentences with embedded a subject extraction, like Who did the director announce nominated him? which does not allow that, due to the constraint known as the that-trace constraint; see Pesetsky (2017) for a recent overview). Those trials were categorized as non-

scorable. Responses that did not contain any filler-gap dependency at all were 
also categorized as non-scorable. In addition, one item (item \#6 in Appendix B) was excluded from analyses because it contained an error.

\subsection{Results}

In Experiment 3, $11.0 \%$ of the trials (203 out of 1840 trials) were identified as non-scorable, so they were excluded from subsequent analyses. The rates of non-scorable responses across four conditions were as follows: Null-Different: 13.9\%; Null-Same: 8.7\%; That-Different: 10.7\%; That-Same: $10.9 \%$. The rate of non-scorable responses were higher than in Experiments 1 and 2 probably because responses that did not preserve the relevant structure of the original sentences were identified as non-scorable, and perhaps also because sentences with cross-clausal filler-gap dependencies were harder to memorize and produce.

The rates of that production across conditions is summarized in Figure 14. Table 4 reports the statistical results. In the different verb condition, speakers on average produced that $36.7 \%$ of the time given null complementizer primes. In contrast, speakers on average produced that $56.1 \%$ of the time given that primes. That is, there was a $19.4 \%$ structural priming effect when clause-taking verbs were not repeated. In the same verb condition, speakers produced that $29.7 \%$ of the time given null complementizer primes. In contrast, speakers produced that $64.4 \%$ of the time given that primes. That is, there was a $34.7 \%$ structural priming effect when clause-taking verbs were repeated. This means that the structural priming effect was overall $15.3 \%$ bigger when clause-taking verbs were repeated than when they were not. Reflecting this pattern, there was evidence for the two-way interaction between Prime Type and Verb Type, and this two-way interaction did not interact with Target Type (i.e., there was no three-way interaction). There was also evidence for the main effect of Prime Type. There was evidence for the interaction between Prime Type and Target Type, suggesting that the speakers showed a larger priming effect when original sentences did not contain that, as the joint analysis of Experiments 1 and 2 also suggested. Finally, there was evidence for the main effect of Target Type, simply suggesting that speakers had some memory of whether the original target 
sentences contained that or not. There was no evidence for other effects.

To assess if the current data provides evidence for the lexically independent complementizer priming effect, a nested model evaluated the effect of Prime Type within each level of Verb Type. This analysis revealed evidence for the effect of Prime Type in the different verb condition $(\hat{\beta}=1.26,95 \% \mathrm{CrI}=$ $[0.84,1.68], \operatorname{Pr}(\beta>0)>0.99)$ and in the same verb condition $(\hat{\beta}=2.01$, $95 \% \mathrm{CrI}=[1.59,2.44], \operatorname{Pr}(\beta>0)>0.99)$, suggesting that there was lexically independent priming of that in Experiment 3 just like in Experiments 1 and 2, but the effect was bigger in the same verb condition as shown by the interaction between Prime Type and Verb Type.

Table 4: The statistical results in Experiment 3. $95 \%$ credible intervals that did not include zero are bold-faced. The R-style model formula was: that PrimeType*VerbType*TargetType + (1+PrimeType*VerbType | Subject $)+(1+$ PrimeType*VerbType | Item)

\begin{tabular}{llll}
\hline Term & Estimate & Est. Error & $95 \%$ CrI \\
\hline Intercept & -0.20 & 0.19 & {$[-0.59,0.18]$} \\
Prime Type & 1.66 & 0.17 & {$[\mathbf{1 . 3 2}, \mathbf{2 . 0 1}]$} \\
Verb Type & 0.04 & 0.15 & {$[-0.25,0.34]$} \\
Target Type & 1.75 & 0.28 & {$[\mathbf{1 . 2 0}, \mathbf{2 . 3 1}]$} \\
Prime Type x Verb Type & 0.78 & 0.28 & {$[\mathbf{0 . 2 4}, \mathbf{1 . 3 3}]$} \\
Prime Type x Target Type & -0.78 & 0.28 & {$[\mathbf{- 1 . 3 3}, \mathbf{- 0 . 2 2}]$} \\
Verb Type x Target Type & -0.11 & 0.28 & {$[-0.66,0.43]$} \\
Prime Type x Verb Type x Target Type & 0.05 & 0.52 & {$[-0.98,1.07]$} \\
\hline
\end{tabular}

\subsection{Discussion}

The lexical boost effect for that-priming was observed in Experiment 1, where both prime and target sentences contained no cross-clausal filler-gap dependencies, but not in Experiment 2, where only prime sentences contained cross-clausal filler-gap dependencies. This contrast was predicted by the TAGbased model, but alternative explanations are possible. In particular, the lack of the lexical boost effect in Experiment 2 may have been due to the increased complexity of prime sentences. Experiment 3 showed that when both prime 
Figure 14: The by-subject mean percentage that production across conditions in Experiment 3. The left panel shows the data from trials where target sentences contained no that. The middle panel shows the data from trials where target sentences contained that. The right panel shows the data from all trials. The error bars represent the by-subject standard error of the means.

\section{Experiment $3(\mathrm{FG}-\mathrm{FG})$}

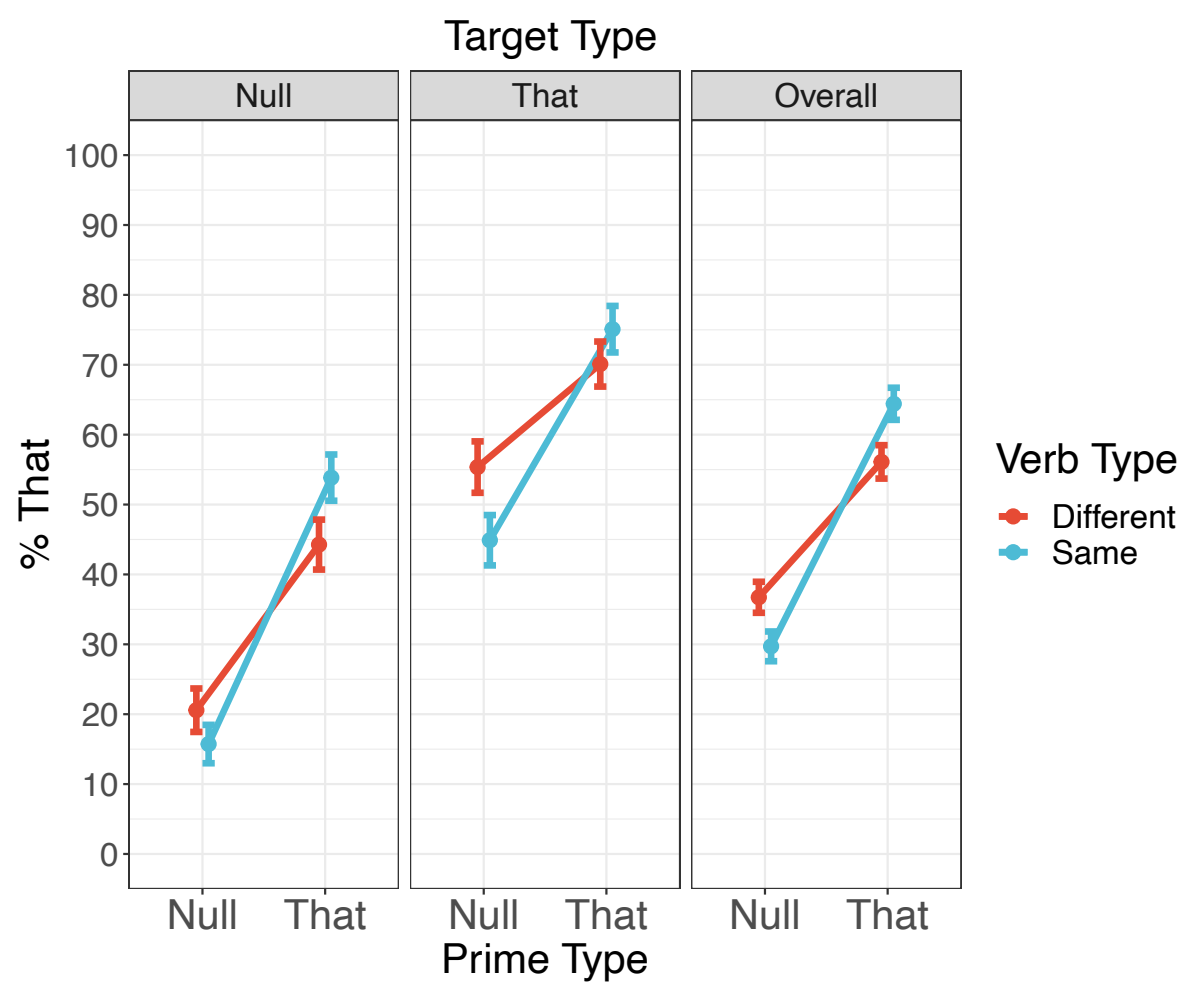

and target sentences contained a cross-clausal filler-gap dependency, the lexical boost effect for that-priming was observed. This result is difficult to account for based on the hypothesis that the lack of the lexical boost effect in Experiment 2 was due to the increased complexity of prime sentences. In Experiment 3, prime sentences had the same level of complexity as in Experiment 2.

There are at least two alternative accounts of why the lexical boost effect was selectively observed in Experiments 1 and 3, and not in Experiment 2. First, the lexeme-based bigram account can still at least partially be maintained. In 
Experiments 1 and 3, prime and target sentences shared relevant bigrams (e.g., announced that). Meanwhile, in Experiment 2, prime and target sentences did not share relevant bigrams (e.g., prime sentences had strings like announce that and the target sentences had strings like announced that). Thus, it may still be the case that the lexical boost effect reflects the priming of lexeme-level bigrams.

Second, the selective lexical boost effect may reflect superficial similarities between prime and target sentences. For example, the lexical boost effect may occur only when target and prime sentences are both declarative sentences (as in Experiment 1) or wh-questions containing who (as in Experiment 3). It is unclear mechanistically how this conditioning of the lexical boost effect arises, but prime and target sentences may have to be sufficiently similar for the lexical boost effect to occur. This account is distinct from the TAG-based model. In the TAG-based model, what is relevant is not whether both prime and target sentences are superficially similar, but whether both prime and target sentences share relevant elementary trees. Experiment 4 aimed to eliminate the possibility that the lexical boost effect occurs only when prime and target sentences are wh-questions.

In Experiment 3, the rate of that production was overall lower than in Experiments 1 and 2. This is not surprising, given the previous evidence that speakers are less likely to use that in the presence of cross-clausal filler-gap dependencies in naturalistic production (Phillips (2013); Chacón (2015)) and production in experimental settings (Momma (2021); McDaniel et al. (2015)).

Finally, in Experiment 1, repeating clause-taking verbs primarily enhanced the priming of the that complementizer. Meanwhile, in Experiment 3, it enhanced the priming of the that complementizer and the null complementizer, leading to an overall larger lexical boost effect. This potential difference may be because elementary trees for sentences with cross-clausal filler-gap dependencies are less frequent than elementary trees for sentences without cross-clausal filler-gap dependencies. If the magnitude of elementary tree priming is larger for low-frequency elementary trees (cf. the inverse preference effect; Jaeger \& Snider (2013); Pickering \& Ferreira (2008); Kaschak \& Borreggine (2008)), the 
lexical boost effect is expected to be larger when prime sentences involve crossclausal filler-gap dependencies (as in Experiment 3) than when they do not (as in Experiment 1).

\section{Experiment 4}

Experiment 3 showed that when both prime and target sentences contained cross-clausal filler-gap dependencies, repeating clause-taking verbs increased the magnitude of the that-priming effect. This finding ruled out the possibility that the absence of the lexical boost effect in Experiment 2 was due to the increased complexity of prime sentences. However, it may still be the case that the lexical boost effect only occurs when both prime and target sentences are superficially similar wh-questions (Experiment 3) or when both are declarative sentences with no wh-phrases in them (Experiment 1). Experiment 4 evaluated this possibility by changing prime sentences in Experiment 3 to matrix subject wh-questions, like in the following:

(5) Who announced/implied (that) he would promote the manager? [Prime]

The target sentences in Experiment 4 were identical to those in Experiment 3. Sentences like (5) are wh-questions, but because wh-phrases do not move across the complementizer structure, the elementary trees headed by clausetaking verbs are not shared with sentences with cross-clausal filler-gap dependencies. Therefore, if the lexical boost effect again fails to be observed in Experiment 4, Experiment 4 would provide evidence against the possibility that the absence of the lexical boost effect in Experiment 2 was simply due to prime sentences being wh-questions and target sentences being declarative sentences.

\subsection{Method}

\subsubsection{Participants}

Eighty monolingual English speakers were recruited using Prolific Academic. Five participants were replaced due to their failure to follow the instruction or because they produced one or no scorable response in one or more conditions. 


\subsubsection{Materials}

Prime sentences in Experiment 4 were constructed by modifying prime sentences from Experiment 3 to matrix subject wh-questions. The target sentences were identical to those in Experiment 3.

\subsubsection{Procedure, scoring and analyses}

The experimental and analysis procedures were the same as in Experiments 1,2 and 3.

\subsection{Results}

In Experiment 4, $15.0 \%$ of the trials (287 out of 1920 trials) were identified as non-scorable, so they were excluded from the subsequent analyses. The rates of non-scorable responses across four conditions were as follows: Null-Different: 16.5\%; Null-Same: 13.3\%; That-Different: 14.8\%; That-Same: $15.2 \%$.

The rates of that production across conditions are summarized in Figure 15. Table 5 reports the statistical results. In the different verb condition, speakers on average produced that $29.6 \%$ of the time given null complementizer primes. In contrast, speakers on average produced that $45.1 \%$ of the time given that primes. That is, there was a $15.5 \%$ structural priming effect when clause-taking verbs were not repeated. In the same verb condition, speakers produced that $32.4 \%$ of the time given null complementizer primes. In contrast, speakers produced that $46.9 \%$ of the time given that primes. That is, there was a $14.5 \%$ structural priming effect when clause-taking verbs were repeated. This means that the structural priming effect was overall $1.00 \%$ smaller when clause-taking verbs were repeated than when they were not repeated. Reflecting this pattern, there was no evidence for the two-way interaction between Prime Type and Verb Type. There was clear evidence for the main effect of Prime Type. Finally, there was evidence for the main effect of Target Type, simply suggesting that speakers had some memory of whether original sentences contained that or not. There was no evidence for the other effects. 
Figure 15: The by-subject mean percentage that production across conditions in Experiment 4. The left panel shows the data from trials where target sentences contained no that. The middle panel shows the data from trials where target sentences contained that. The right panel shows the data from all trials. The error bars represent the by-subject standard error of the means.

\section{Experiment 4 (noFG-FG)}

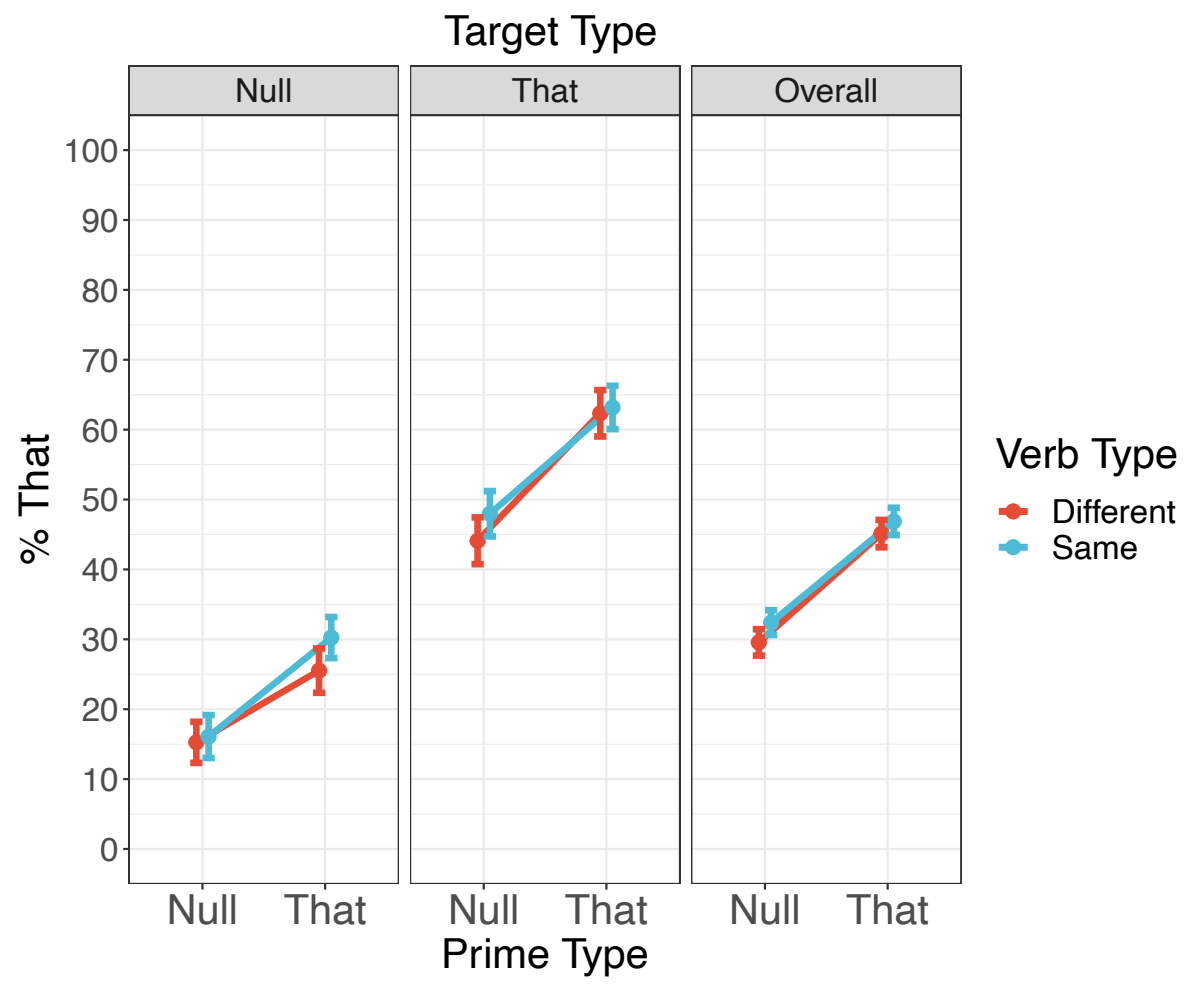

Like in Experiments 1-3, a nested model evaluated the effect of Prime Type within each level of Verb Type. This analysis revealed evidence for the effect of Prime Type in the different verb condition $(\hat{\beta}=1.01,95 \% \mathrm{CrI}=[0.57,1.45]$, $\operatorname{Pr}(\beta>0)>0.99)$ and in the same verb condition $(\hat{\beta}=1.04,95 \% \mathrm{CrI}=$ $[0.62,1.46], \operatorname{Pr}(\beta>0)>0.99)$.

\subsection{Discussion}

Experiment 4 showed that the lexical boost effect was absent when only target sentences contained a cross-clausal filler-gap dependency. In Experiment 4, 
Table 5: The statistical results in Experiment 4. 95\% credible intervals that did not include zero are bold-faced. The R-style model formula was: that $\sim$ PrimeType*VerbType*TargetType + $(1+$ PrimeType*VerbType $\mid$ Subject $)+(1+$ PrimeType*VerbType | Item)

\begin{tabular}{llll}
\hline Term & Estimate & Est. Error & $95 \%$ CrI \\
\hline Intercept & -0.81 & 0.24 & {$[-1.30,-0.34]$} \\
Prime Type & 1.05 & 0.17 & {$[\mathbf{0 . 7 1}, \mathbf{1 . 3 9}]$} \\
Verb Type & 0.11 & 0.19 & {$[-0.27,0.47]$} \\
Target Type & 2.28 & 0.30 & {$[\mathbf{1 . 6 9}, \mathbf{2 . 8 8}]$} \\
Prime Type x Verb Type & 0.05 & 0.34 & {$[-0.62,0.71]$} \\
Prime Type x Target Type & 0.08 & 0.32 & {$[-0.55,0.71]$} \\
Verb Type x Target Type & 0.01 & 0.36 & {$[-0.69,0.71]$} \\
Prime Type x Verb Type x Target Type & -0.46 & 0.63 & {$[-1.71,0.77]$} \\
\hline
\end{tabular}

both prime and target sentences were superficially similar wh-questions. Prime sentences in Experiment 4 contained filler-gap dependencies. They just did not cross the complementizer structure. Despite this superficial similarity between prime and target sentences, the results were different from Experiment 3, where the lexical boost effect was observed (see the result section of Experiment 5 for the statistical analysis comparing the magnitude of the lexical boost effect across Experiments 3-5). This means that the superficial similarity between prime and target sentences was not sufficient to trigger the lexical boost effect, reinforcing the claim that the lexical boost effect for that-priming occurs specifically when elementary trees headed by clause-taking verbs are repeated.

\section{Experiment 5}

Experiment 4 showed that when only target sentences contained a crossclausal filler-gap dependency, repeating clause-taking verbs did not increase the magnitude of the that-priming effect. This was so despite prime and target sentences being superficially similar wh-questions. However, it may still be that the lexical boost effect occurs only when repeated verbs share the same inflectional forms, that is, when prime and target sentences share a lexeme level bigram (e.g., announced that). This possibility is not a priori likely, because a past 
study found that the lexical boost effect for dative priming was not modulated by whether verbs of prime and target sentences share the same inflectional form (Pickering \& Branigan (1998)). However, there is no guarantee that the dative priming effect and the that-priming effect behave similarly in this regard. To evaluate this possibility, in Experiment 5, target sentences contained crossclausal filler-gap dependencies, but in an indirect question context:

(6) I wonder who the director announced (that) he would nominate? [Target]

\subsection{Method}

\subsubsection{Participants}

Ninety monolingual English speakers were recruited using Prolific Academic. Eight participants were replaced due to their failure to follow the instruction or because they produced one or no scorable response in one or more conditions.

\subsubsection{Materials}

Prime sentences for Experiment 5 were constructed by modifying the target sentences from Experiments 3 and 4 to embedded object wh-questions. Prime sentences were identical to those in Experiments 3 and 4.

\subsubsection{Procedure, scoring and analyses}

The experimental and analysis procedures were the same as in Experiments $1-4$.

\subsection{Results}

In Experiment 5, $16.7 \%$ of the trials (360 out of 2160 trials) were identified as non-scorable, so they were excluded from subsequent analyses. The rates of non-scorable responses across four conditions were as follows: Null-Different: 19.6\%; Null-Same: 14.8\%; That-Different: 17.2\%; That-Same: $15.0 \%$.

The rates of that production across conditions are summarized in Figure 16. Table 6 reports the statistical results. In the different verb condition, speakers on 
average produced that $23.4 \%$ of the time given null complementizer primes. In contrast, speakers on average produced that $36.9 \%$ of the time given that primes. That is, there was a $13.5 \%$ structural priming effect when clause-taking verbs were not repeated. In the same verb condition, speakers produced that $22.6 \%$ of the time given null complementizer primes. In contrast, speakers produced that $34.4 \%$ of the time given that primes. That is, there was an $11.8 \%$ structural priming effect when clause-taking verbs were repeated. This means that the structural priming effect was overall $1.7 \%$ smaller when the matrix verbs were repeated than when they were not repeated. Reflecting this pattern, there was no evidence for the two-way interaction between Prime Type and Verb Type. There was clear evidence for the main effect of Prime Type. Finally, there was evidence for the main effect of Target Type, simply suggesting that speakers had some memory of whether original target sentences contained that or not. There was no evidence for the presence of other effects.

Like in Experiments 1-4, a nested model evaluated the effect of Prime Type within each level of Verb Type. This analysis revealed evidence for the effect of Prime Type in the different verb condition $(\hat{\beta}=1.02,95 \% \mathrm{CrI}=[0.55,1.51]$, $\operatorname{Pr}(\beta>0)>0.99)$ and in the same verb condition $(\hat{\beta}=1.05,95 \% \mathrm{CrI}=$ $[0.60,1.52], \operatorname{Pr}(\beta>0)>0.99)$.

Finally, a model directly compared the magnitude of the lexical boost effect in Experiments 3-5. The fixed effects of this model included Prime Type, Verb Type, Target Type, Experiment, and their interactions. The random-effects structure included by-subject and by-item random intercepts, by-subject random slopes for Prime Type, Verb Type, and their interactions, and by-item random slopes for Prime Type, Verb Type, Experiment, and their interactions. The factor Experiment had the hypothesis matrix where Experiment 3 was compared against the grand mean (Experiments 3, 4, and 5 were coded as 2/3, $-1 / 3,-1 / 3$ respectively in the first contrast of Experiment), and Experiment 5 was compared against the grand mean (Experiments 3, 4 and 5 were coded as $-1 / 3,-1 / 3,2 / 3$ respectively in the second contrast of Experiment). To generate the appropriate contrast matrix corresponding to this non-orthogonal hypothe- 
sis matrix, the generalized inverse method was applied, as described by Schad et al. (2020). The first contrast tested the critical hypothesis that the lexical boost effect is stronger when both prime and target sentences contained cross-clausal filler-gap dependencies (as in Experiment 3). The second contrast tested the hypothesis that the lexical boost effect occurs only when relevant verbs share the same inflectional form (as in Experiment 5). The results of this analysis are summarized in Table 7. Most relevantly, there was evidence for the three-way interaction between Prime Type, Verb Type, and the first contrast of Experiment, suggesting that the magnitude of the lexical boost effect was larger in Experiment 3. Aside from the critical three-way interaction, there was evidence for the main effects of Prime Type, Target Type, and Experiment. I avoid interpreting these main effects because there was evidence for interactions involving each of those factors. There was also evidence for the two-way interaction between Target Type and Experiment, but again, given the evidence for the higher-order interaction, I avoid giving an interpretation to this effect. Finally, there was evidence for the two-way interaction between Prime Type and Target Type, suggesting that the priming effect is bigger when memorized target sentences did not contain that.

Table 6: The statistical results in Experiment 5. $95 \%$ credible intervals that did not include zero are bold-faced. The R-style model formula was: that PrimeType*VerbType*TargetType $+(1+$ PrimeType*VerbType $\mid$ Subject $)+(1+$ PrimeType*VerbType | Item)

\begin{tabular}{llll}
\hline Term & Estimate & Est. Error & $95 \%$ CrI \\
\hline Intercept & -1.46 & 0.24 & {$[-1.94,-0.98]$} \\
Prime Type & 1.06 & 0.20 & {$[\mathbf{0 . 6 7}, \mathbf{1 . 4 6}]$} \\
Verb Type & -0.27 & 0.18 & {$[-0.64,0.08]$} \\
Target Type & 1.88 & 0.34 & {$[\mathbf{1 . 2 1}, \mathbf{2 . 5 4}]$} \\
Prime Type x Verb Type & 0.00 & 0.35 & {$[-0.69,0.68]$} \\
Prime Type x Target Type & -0.68 & 0.36 & {$[-1.38,0.03]$} \\
Verb Type x Target Type & 0.49 & 0.32 & {$[-0.14,1.13]$} \\
Prime Type x Verb Type x Target Type & 0.64 & 0.63 & {$[-0.60,1.86]$} \\
\hline
\end{tabular}


Table 7: The results of the joint analyses of Experiments 3-5. 95\% credible intervals that did not include zero are bold-faced. The R-style model formula was: that $\sim$ PrimeType*VerbRepetition*TargetType*Experiment + (1+PrimeType*VerbRepetition | Subject) $+(1+$ PrimeType*VerbRepetition*Experiment $\mid$ Item $)$

\begin{tabular}{llll}
\hline Term & Estimate & Est. Error & $95 \%$ CrI \\
\hline Intercept & -0.81 & 0.16 & {$[-\mathbf{1 . 1 4}, \mathbf{- 0 . 4 9}]$} \\
Prime Type & 1.25 & 0.11 & {$[\mathbf{1 . 0 4}, \mathbf{1 . 4 6}]$} \\
Verb Type & -0.02 & 0.09 & {$[-0.21,0.16]$} \\
Exp. 3 & 0.61 & 0.15 & {$[\mathbf{0 . 3 2}, \mathbf{0 . 9 0}]$} \\
Exp. 5 & -0.62 & 0.15 & {$[-\mathbf{0 . 9 2}, \mathbf{- 0 . 3 4}]$} \\
Target Type & 1.95 & 0.27 & {$[\mathbf{1 . 4 1 , 2 . 4 7}]$} \\
Prime Type x Verb Type & 0.29 & 0.17 & {$[-0.06,0.62]$} \\
Prime Type x Exp. 3 & 0.44 & 0.13 & {$[\mathbf{0 . 1 8}, \mathbf{0 . 7 0}]$} \\
Prime Type x Exp. 5 & -0.22 & 0.14 & {$[-0.49,0.05]$} \\
Verb Type x Exp. 3 & 0.06 & 0.13 & {$[-0.19,0.31]$} \\
Verb Type x Exp. 5 & -0.19 & 0.13 & {$[-\mathbf{0 . 4 5}, \mathbf{0 . 0 6}]$} \\
Prime Type x Target Type & -0.45 & 0.19 & {$[-\mathbf{0 . 8 3}, \mathbf{- 0 . 0 6}]$} \\
Verb Type x Target Type & 0.15 & 0.18 & {$[-0.20,0.50]$} \\
Exp. 3 x Target Type & -0.19 & 0.13 & {$[-0.44,0.06]$} \\
Exp. 5 x Target Type & -0.09 & 0.15 & {$[-0.37,0.20]$} \\
Prime Type x Verb Type x Exp. 3 & 0.55 & 0.23 & {$[\mathbf{0 . 0 9}, \mathbf{1 . 0 1}]$} \\
Prime Type x Verb Type x Exp. 5 & -0.31 & 0.30 & {$[-0.88,0.27]$} \\
Prime Type x Verb Type x Target Type & 0.08 & 0.33 & {$[-0.55,0.73]$} \\
Prime Type x Exp. 3 x Target Type & -0.28 & 0.23 & {$[-0.74,0.18]$} \\
Prime Type x Exp. 5 x Target Type & -0.22 & 0.26 & {$[-0.71,0.29]$} \\
Verb Type x Exp. 3 x Target Type & -0.18 & 0.24 & {$[-0.66,0.30]$} \\
Verb Type x Exp. 5 x Target Type & 0.31 & 0.25 & {$[-0.17,0.80]$} \\
Prime Type x Verb Type x Target Type x Exp. 3 3 & -0.05 & 0.45 & {$[-0.93,0.83]$} \\
Prime Type x Verb Type x Target Type x Exp. 5 & 0.59 & 0.56 & {$[-0.53,1.70]$} \\
\hline & & & \\
\hline & &
\end{tabular}


Figure 16: The by-subject mean percentage that production across conditions in Experiment 5. The left panel shows the data from trials where target sentences contained no that. The middle panel shows the data from trials where target sentences contained that. The right panel shows the data from all trials. The error bars represent the by-subject standard error of the means.

\section{Experiment 5 (noFG-FG)}

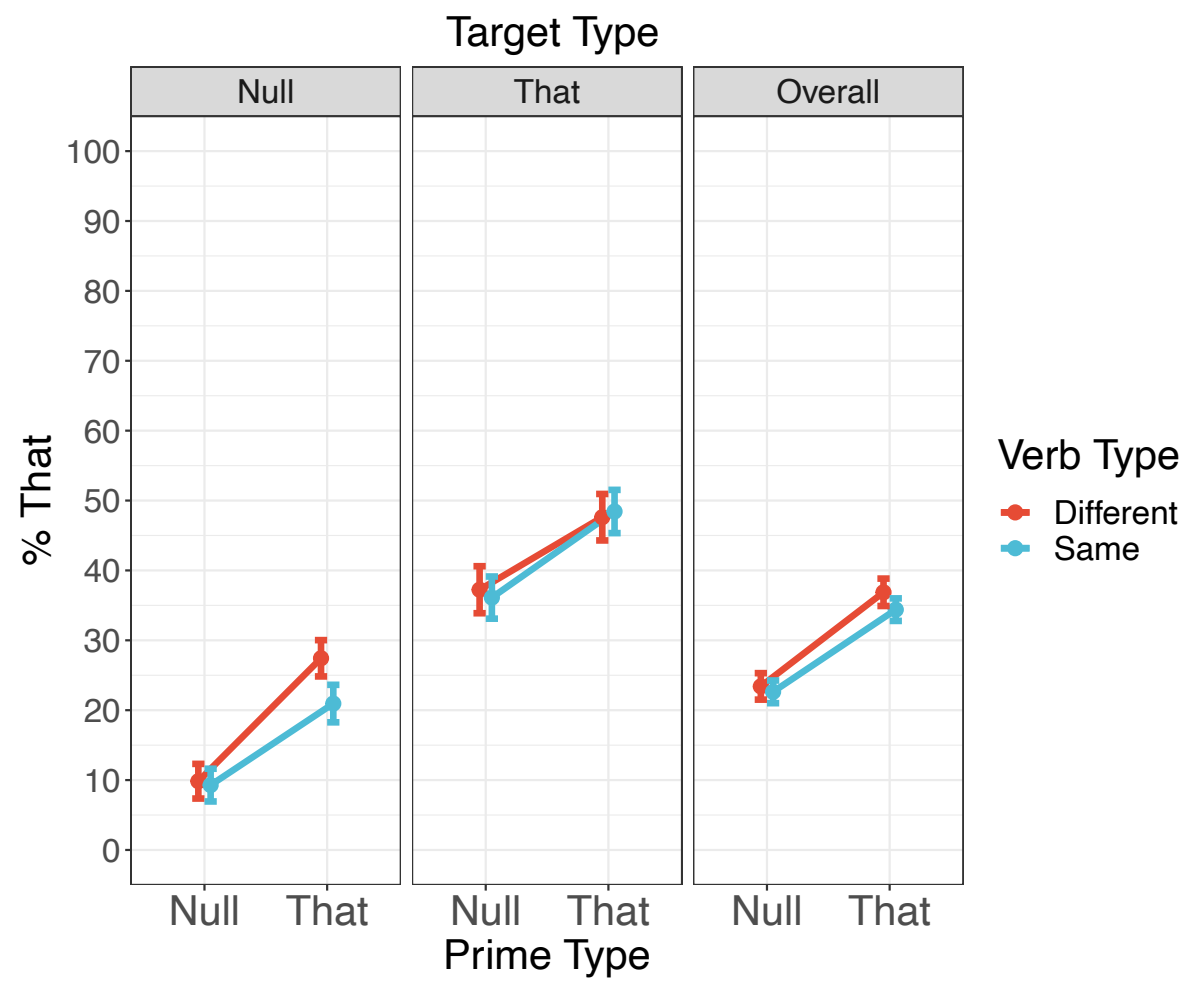

\subsection{Discussion}

In Experiment 5, target sentences were embedded wh-questions, while prime sentences were declarative sentences with no wh-phrases. Critically, because target sentences were embedded wh-questions, the inflectional form of relevant clause-taking verbs was shared across prime and target sentences. Despite this, the lexical boost effect was not observed in Experiment 5, unlike in Experiment 3. Thus, the concern that the lexical boost effect in Experiments 1 and 3 was due to the priming of lexeme level bigram is empirically unwarranted, because 
that view would predict the lexical boost effect in Experiment 5, contrary to the results. The TAG-based model correctly predicts that the inflectional form of clause-taking verbs should not matter.

The joint analyses of the data from Experiments 3-5 also provided evidence that the magnitude of the lexical boost effect was indeed larger in Experiment 3 , as indicated by the three-way interaction between Prime Type, Verb Type, and the first contrast of Experiment. Thus, Experiments 3-5 together provided evidence for the TAG-based model's prediction that the lexical boost effect should be selectively observed when both prime and target sentences contained cross-clausal filler-gap dependencies, as in Experiment 3. The surface similarity between sentences (prime and target being both wh-questions) and the shared inflectional form of clause-taking verbs (Experiment 5) was not sufficient to explain the current results.

\section{General Discussion}

In the current study, a simple computational model of structural priming and its lexical boost effects based on the TAG framework was presented. This model was motivated by past findings by Momma (2021) and it predicted that the lexical boost effect for that-priming should be observed selectively when both prime and target sentences share relevant elementary trees, that is, when both prime and target sentences contained cross-clausal filler-gap dependencies or when neither does. Confirming this prediction, five experiments together showed that the lexical boost effect for complementizer priming was observed selectively when both target and prime sentences contained cross-clausal fillergap dependencies (Experiment 3) or when neither did (Experiment 1). When

only prime sentences (Experiment 2) or target sentences (Experiments 4 and 5) contained cross-clausal filler-gap dependencies, the lexical boost effect did not occur. Figure 17 shows the magnitude of the lexical boost effect across experiments. This pattern confirmed the prediction of the TAG-based model. As far as I am aware, no existing production models make this prediction. 
Figure 17: The lexical boost effect across experiments. Error bars represent by-subject standard errors.

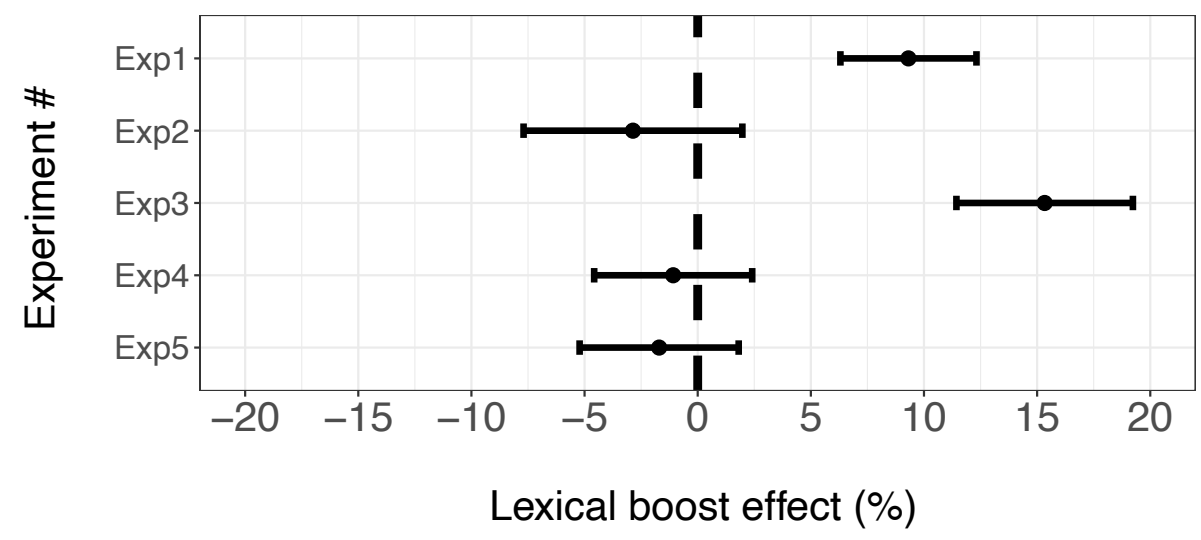

\subsection{Implications on theories of sentence production}

The current study corroborated the TAG-based model. This model has several properties that are not found in currently dominant theories like Levelt (1989), Bock \& Levelt (1994) and Bock \& Ferreira (2013). First, the current model claims that filler-gap dependencies are established first and intervening material is adjoined later. This departs from the usually held, though often implicit, assumption that sentence planning proceeds from "beginning-to-end" (Dell et al., 2008; Ferreira et al., 2018). Rather, under the current view, sentence planning may be shaped by hierarchically-defined grammatical dependencies in a top-down fashion, over and above word order. This view is compatible with a class of production models known as the hierarchically incremental view (e.g., Bock et al. (2003); Bock \& Ferreira (2013); Lee et al. (2013)), though that view is compatible with a variety of production models including the ones that assume that sentence planning proceeds from "beginning-to-end", and the current proposals make claims that go above and beyond the general framework offered by that view.

Second, the current model also claims that clause-taking verbs like think head two distinct structures, one participating in substitution (for sentences 
without cross-clausal filler-gap dependencies) and the other participating in adjoining (for sentences with cross-clausal filler-gap dependencies). To the extent the current results support the TAG-based model, they suggest that speakers (and comprehenders, see below) use these two distinct compositional processes and two distinct structural representations needed for each type of composition.

Third, the current model assumes two levels of structural representations, one level encoding nodes of elementary trees and the other level encoding elementary trees themselves. This idea resembles previous proposals about the representations of idioms (Sprenger et al., 2006) and particle verbs (Chen \& Hartsuiker, 2021), where complex representations are separately represented from their parts (cf. super lemma). The current proposal is that this twolayer architecture is not restricted to idioms and particle verbs. The current idea is also similar to the architecture of the phonological encoding aspects of models like Levelt et al. (1999) where different layers encode the same level of representations with different grain sizes. For example, in Levelt et al. (1999), the lexeme level encodes holistic lexical forms, and nodes in the lexeme level are connected to nodes representing their parts (phonemes). Thus, if the current TAG-based model is right, syntactic and phonological levels of processing in production may share similar architectures, in that there is a layer representing a complex whole, another layer representing its parts, and connections representing part-whole relations.

Finally, the current model claims that the lexically independent and lexically dependent components of structural priming are due to two distinct underlying mechanisms. This view is not new, and is shared with many previous proposals such as Pickering \& Branigan (1998), Hartsuiker et al. (2008), Reitter et al. (2011), and Chang et al. (2006), although details are different. The current model claims that lexically independent structural priming arises from the strengthening of the connection between a relevant conceptual representation (e.g., GOAL and some conceptual features associated with complement clauses represented by the $\mathrm{X}$ node) and a subtree level node (e.g., appl and that), while the lexical boost effect arises from residual activation of an elementary tree 
representation. Thus, one contribution of the current study is to offer a new implementation of the familiar idea that structural priming has (at least) two sources.

\subsection{The nature of lexical boost effect}

The current study challenges the claim that the lexical boost effect is due to the explicit memory of prime sentences (Chang et al., 2006), and relatedly, the claim that it is due to spreading activation from any words surrounding the primed structures (Reitter et al., 2011). These accounts predict that the lexical boost effect should be observed in all experiments in the current study, contrary to the results.

The current results also contrast with the findings by Scheepers et al. (2017) that repeating any content word leads to the lexical boost effect, which has been taken as consistent with Chang et al. (2006) and Reitter et al. (2011). They are instead more in line with the study by Carminati et al. (2019) and van Gompel et al. (2022). The task that Scheepers et al. (2017) used was different from the current study, so direct comparisons need to be done carefully. However, a more recent study (Carminati et al., 2019) failed to replicate the finding by Scheepers et al. (2017) in multiple experiments, even when the same task was used, and van Gompel et al. (2022) suggested that the lexical boost effect from the repetition of subject nouns has a strategic origin. Thus, together with Carminati et al. (2019) and van Gompel et al. (2022), the current study challenges the empirical grounds for the explicit memory and the spreading activation accounts of the

lexical boost effect (see also Yan et al. (2018) for the neuropsychological evidence against the explicit memory account). This does not mean that the lexical boost effect can be used uncritically to make inferences about the relationship between structural and lexical representations. However, when the lexical boost effect is shown to be selective to specific contexts, as in the current experiments, it provides reasonable evidence that only certain types of words hold primable relation to certain structures.

More concretely, the current model claims that the lexical boost effect can be 
viewed as priming of elementary trees in TAG. Via simulation, it was shown that lexically independent and lexically dependent components of structural priming can be coherently captured under the TAG framework. This claim captures the lexical boost effect, and as the current study shows, it yields restrictive predictions about when the lexical boost effect should occur. However, there are some challenges to the current view. For example, the lexical boost effect due to the repetition of a head noun reported in Cleland \& Pickering (2003) is difficult to capture straightforwardly under the current proposal. In Cleland \& Pickering (2003), it was found that repeating a head noun, but not an adjective, enhances the priming of prenominal adjective (e.g., the green table) vs. postnominal relative clause (e.g., the table that is green) modifications. In TAG, both prenominal adjectives and post-nominal relative clauses are added by adjoining and adjoined structures do not contain nominal heads. Thus, if the lexical boost effect is identified as the priming of elementary trees, the results from Cleland \& Pickering (2003) cannot easily be captured.

One potential solution to this problem is to assume excitatory connections between noun lemmas and the relative clause complementizer (or some other functional head associated with relative clauses) in the subtree level layer. The lexical boost effect reported by Cleland \& Pickering (2003) could be due to prime sentences strengthening this connection. This approach is essentially similar to the original explanation of the lexical boost effect offered by Pickering \& Branigan (1998). Pickering \& Branigan (1998) suggested that the lexical boost effect for dative priming arises from residual activation of a connection between a combinatorial node and a verb lemma. The current modeling approach does not assume combinatorial nodes (see Introduction), and it does not have any way of representing the activation of a connection (because connections do not have activation in the standard connectionist-style modeling framework). Thus, I instead posit that the lexical boost effect reported by Cleland \& Pickering (2003) arises from the strengthening of the connection between a noun lemma and the relative clause complementizer. However, I should acknowledge that adopting this solution amounts to positing that the lexical boost effect reported 
by Cleland \& Pickering (2003) and the lexical boost effect reported in the current study have different mechanisms.

An additional challenge for the current TAG-based approach comes from Scheepers (2003). Scheepers (2003) found that, in German, the attachment height of relative clauses in expressions like the servant of the actress who... can be primed. This priming effect cannot be explained by the strengthening of the connection between a concept level node and a subtree level node or by the residual activation of an elementary tree node. The reason is that two possible options are generated by the identical set of elementary trees. Scheepers (2003) interpreted this priming effect as priming of the order of phrase-structure rule applications. However, this explanation does not translate straightforwardly to TAG terms because, in TAG, the order of combinatorial operations does not determine attachment heights. For instance, the auxiliary tree for of the actress can adjoin to the DP elementary tree the servant first, and the auxiliary tree for who can then adjoin to the higher N' node of the derived structure to yield the structure corresponding to the high-attachment reading. Alternatively, the auxiliary tree for of the actress can adjoin to the DP elementary tree the servant first, and the auxiliary tree for who can then adjoin to the lower N' node to yield a derived structure corresponding to the low-attachment reading. In both cases, the order in which elementary trees are combined is identical, and the only difference is which N' node the auxiliary tree for who... adjoins to. Therefore, to explain the priming effect reported by Scheepers (2003) in the TAG framework, it needs to be assumed that speakers and comprehenders somehow retain the abstract (lexically independent) memory of the relative location of the adjoining site. Unfortunately, this essentially amounts to restating the observation in TAG terms. Whether a more principled explanation is possible and whether a unified explanation of various structural priming effects can be attained remain to be an open question. 


\subsection{The relationship between comprehension and production}

The current results may also shed light on the relationship between comprehension and production. In the current experiments, participants read aloud prime sentences. Reading aloud in some sense involves production, but reading aloud starts from form-based inputs, just like in comprehension. Structurebuilding processes in a reading-aloud task are thus arguably similar to those during normal comprehension. In contrast, structure-building processes in sentence recall are arguably similar to those during production, because the sentence recall task likely involves the regeneration of sentences from conceptual representations (Potter \& Lombardi (1990, 1998); McDonald et al. (1993); Ferreira (2003) among others). Under these assumptions, the structural priming effects observed in the current study reflect priming from structural representations built from form-based inputs to structural representations build from conceptual inputs. That is, elementary trees used to build filler-gap dependency structures, as well as the mechanisms for combining them, may be shared between comprehension and production. Indeed, the TAG-based model of fillergap dependency production presented here is abstractly similar to prominent models of filler-gap dependency comprehension, where filler-gap dependencies are processed predictively (Omaki et al., 2015; Stowe, 1986; Aoshima et al., 2004; Frazier \& Flores d'Arcais, 1989; Fodor, 1978). In predictive models of filler-gap dependency comprehension, comprehenders posit the gap representation as soon as the filler is encountered, and the material in between the filler and the gap is integrated after the gap is predicted. In the TAG-based model of filler-gap dependency production, speakers posit the gap representation simultaneously with the filler, and the material in between the filler and the gap is planned after the gap is planned. The primary difference is that speakers, under usual circumstances, can be more certain about the properties of the gap than comprehenders. After all, speakers decide where to put the gap. In comprehension, without more information about the filler, it is not possible to be certain about the grammatical position of the gap. In production, determining the appropriate grammatical status of the gap is possible even before the 
articulation of the filler begins using conceptual inputs. Thus, comprehension and production may share the same structure-building mechanisms for filler-gap dependency formation processes, and differences may reduce to the amount and type of information available at each stage of filler-gap dependency formation processes (cf. Momma \& Phillips (2018)).

\section{Conclusion}

The current study introduced a computational model of structural priming based on the TAG formalism and offered evidence for its prediction that, as far as I am aware, does not follow from any existing models of structural priming or models of sentence production more generally. Although the current results confirm the prediction of the TAG-based model, that does not mean that models based on other frameworks cannot be built to capture the current results. However, regardless of whether the current model is right, adequate theories of sentence production ultimately need to be able to capture filler-gap dependency production. The current study suggests that filler-gap dependency production may involve some processes and mechanisms whose nature may not simply be extrapolated from what we know about the production of simple structures. 


\section{Appendix A. Implementational details of the structural priming model}

The models depicted in Figure 6 and 7 were implemented as interactive activation models. The simulation was run by clamping the activation of one or more of the message level units and letting the activation spread through the network until the activation value of an elementary tree node reached 1 . In the dative priming model, the activation of the GIVE unit and the GOAL unit was clamped at 1 throughout a simulation. In the that-priming model, the activation of the THINK unit, the $\mathrm{X}$ unit (conceptual correlates the presence of an embedded clause), and the Y unit (conceptual correlates of the absence of wh-phrases in an embedded clause) was clamped at 1 throughout a simulation. The Y unit has inhibitory connections to elementary trees containing fillergap dependencies ( $\mathrm{T} 5$ and $\mathrm{T} 6$ ) and auxiliary trees headed by embedding verbs (A1-A4). These inhibitory connections ensured that those elementary trees are selected only when there is some conceptual trigger of a cross-clausal filler-gap dependency. However, when simulating the conditions where target sentences contain cross-clausal filler-gap dependencies, the activation of the $\mathrm{Y}$ node was clamped at 0 throughout a simulation. This allows those elementary trees to be released from inhibition. All excitatory connection weights were by default set to 0.2 and all inhibitory connection weights were set to -0.2 .

The effect of lexically independent structural priming was simulated by increasing the weight of an appropriate connection by 0.005 . Specifically, in the dative priming model, in the DO prime conditions, the connection weight between the GOAL node and the appl node was increased by 0.005 . In the PD priming condition, the connection weight between the GOAL node and the $P P_{\text {give }}$ node was increased by 0.005 . In the that-priming model, in the thatprime condition, the connection weight between the $\mathrm{X}$ node and the that node was increased by 0.005 . Finally, in the null prime condition, the connection weight between the $\mathrm{X}$ node and the null complementizer node was increased by 0.005 .

The effect of lexically dependent structural priming was simulated by in- 
creasing the initial activation level of an appropriate elementary tree node. Specifically, in the dative priming model, in the Same-DO condition, the T1 node had the initial activation of 0.15 (instead of the default 0). In the SamePD condition, the T3 node had the initial activation of 0.15. In the Different-DO condition, the $\mathrm{T} 2$ node had the initial activation of 0.15 . In the Different-PD condition, the T4 node had the initial activation of 0.15 . In the that-priming model, which elementary trees received an initial activation boost depended on whether prime sentences contained cross-clausal filler-gap dependencies. When prime sentences contained no cross-clausal filler-gap dependencies, in the SameThat condition, the T1 node had the initial activation of 0.15. In the Same-Null condition, the T3 node had the initial activation of 0.15. In the Different-That condition, the T2 node had the initial activation of 0.15 . In the Different-Null condition, the T4 node had the initial activation of 0.15 . When prime sentences contained a cross-clausal filler-gap dependency, in the Same- That condition, the A1 node had the initial activation of 0.15. In the Same-Null condition, the A3 node had the initial activation of 0.15 . In the Different- That condition, the A2 node had the initial activation of 0.15 . In the Different-Null condition, the A4 node had the initial activation of 0.15 .

In both models, random noise was added to the connection weights at the beginning of each simulation. The random noise was normally distributed with a mean of 0 and a standard deviation of 0.01 .

In both models, activation spread following the following activation function, bounded by 0 and 1 :

$$
\operatorname{act}_{i}(t)=d * a c t_{i}(t-1)+\sum_{j} w_{i j} * a c t_{j}(t-1)
$$

where $a c t_{i}(t)$ is the activation of the unit in question, $d$ is a decay rate, which is set to 0.8 (the same setting as in Ferreira (1996)), $a_{c} t_{i}(t-1)$ is the activation of the same unit on the previous time step, $w_{i j}$ is the weight to unit $i$ from unit $j$, and $a_{c t}(t-1)$ is the activation of the unit that sends activation to the unit in question on the previous time step. The calculated activation of a unit did not affect the activation of other units until all the units in the model had been 
updated.

At the end of a simulation, an elementary tree is selected. This selected elementary tree was coded as either 0 (the PD response or the null complementizer response) or 1 (the DO response of that response), depending on whether that elementary tree encodes $\mathrm{PD} / \mathrm{DO}$ or the null complementizer/that. To compute the average proportion of DO or that production that can be intuitively compared to human data (as in Figures 9 and 10), in both models, 20 simulations were run for each condition to calculate percentage DO or that production. These 20 simulations can be thought of as repeated trials of a given participant. This procedure was repeated 100 times, that is, 100 "participants" were simulated to calculate the standard errors used in Figures 9 and 10.

\section{Appendix A. Stimulus list}

Prime and target sentences used in Experiment 1. Prime sentences are labeled as (a), and target sentences (b).

1. (a) The manager implied/announced (that) he would promote the employee.

(b) The director announced that the actor would be in the new movie.

2. (a) The activist argued/said (that) the politician should listen to the scientist.

(b) The student said that the teacher was nice.

3. (a) The interviewer suspected/believed (that) the celebrity liked him.

(b) The breeder believed that the dog was well-trained.

4. (a) The detective guessed/heard (that) the victim saw the perpetrator.

(b) The little girl heard that the bird visited her house.

5. (a) The man announced/claimed (that) he married his childhood friend.

(b) The nurse claimed that the patient had fainted. 
6. (a) The scientist claimed/thought (that) the politician should consult him.

(b) The dentist thought that people needed to floss more often.

7. (a) The lawyer feared/suspected (that) her client lied to the judge.

(b) The engineer suspected that the software had a lot of bugs.

8. (a) The teacher assumed/feared (that) the student hated her.

(b) The chef feared that his cake was too sweet.

9. (a) The journalist heard/guessed (that) the politician lied to him.

(b) The engineer guessed that the computer was broken.

10. (a) The attorney believed/argued (that) the witness saw the suspect.

(b) The professor argued that the tuition should be cheaper.

11. (a) The boy said/assumed (that) his sister loved the cat.

(b) The student assumed that the exam was easy.

12. (a) The fortune teller thought/implied (that) the man would marry a famous actress.

(b) The novelist implied that the new character would die.

13. (a) The mayor announced/implied (that) he would appoint her.

(b) The manager implied no one was being laid off.

14. (a) The activist argued/believed (that) the journalist should criticize the politician.

(b) The girl believed the library was closed.

15. (a) The zookeeper thought/said (that) the penguin loved him.

(b) The author said the book sold well.

16. (a) The counselor heard/thought (that) the client feared his father. 
(b) The fireman thought the boy had already been rescued.

17. (a) The queen guessed/heard (that) the knight protected the king.

(b) The doctor heard the patient was angry.

18. (a) The witness believed/claimed (that) the robber attacked the cashier.

(b) The historian claimed the king was well respected.

19. (a) The boss assumed/guessed (that) his employees complained about the customer.

(b) The woman guessed her husband wanted to quit his job.

20. (a) The prosecutor claimed/assumed (that) the man robbed the woman.

(b) The midwife assumed the baby was a boy.

21. (a) The student suspected/feared (that) her roommate would invite her boyfriend to the party.

(b) The author feared his new book would not sell well.

22. (a) The father feared/suspected (that) his son distrusted him.

(b) The farmer suspected the florist was already married.

23. (a) The editor said/argued (that) the journalist should interview the jury.

(b) The union leader argued the employees should be paid more.

24. (a) The king implied/announced (that) the prince would marry the villager's daughter.

(b) The president announced the employees should be paid more.

Prime and target sentences used in Experiment 2.

1. (a) Who did the manager imply/announce (that) he would promote?

(b) The director announced that the actor would be in the new movie. 
2. (a) Who did the activist say/argue (that) the politician should listen to?

(b) The student said that the teacher was nice.

3. (a) Who did the interviewer believe/suspect (that) the celebrity liked?

(b) The breeder believed that the dog was well-trained.

4. (a) Who did the detective guess/hear (that) the victim saw?

(b) The little girl heard that the bird visited her house.

5. (a) Who did the man announce/claim (that) he married?

(b) The nurse claimed that the patient had fainted.

6. (a) Who did the scientist think/claim (that) that the politician should consult?

(b) The dentist asserted that people needed to floss more often.

7. (a) Who did the lawyer suspect/fear (that) her client lied to?

(b) The engineer suspected that the software had a lot of bugs.

8. (a) Who did the teacher assume/fear (that) the student hated?

(b) The chef feared that his cake was too sweet.

9. (a) Who did the journalist hear/guess (that) the politician lied to?

(b) The engineer guessed that the computer was broken.

10. (a) Who did the attorney argue/believe (that) the witness saw?

(b) The professor argued that the tuition should be cheaper.

11. (a) Who did the boy assume/say (that) his sister loved?

(b) The student assumed that the exam was easy.

12. (a) Who did the fortune teller think/imply (that) the man would marry?

(b) The novelist implied that the new character would die.

13. (a) Who did the mayor announce/imply (that) he would appoint? 
(b) The manager implied no one was being laid off.

14. (a) Who did the activist believe/argue (that) the journalist should criticize?

(b) The girl believed the library was closed.

15. (a) Who did the zookeeper say/think (that) the penguin loved?

(b) The author said the book sold well.

16. (a) Who did the counselor hear/think (that) the client feared?

(b) The fireman thought the boy had already been rescued.

17. (a) Who did the queen guess/hear (that) the knight protected?

(b) The doctor heard the patient was angry.

18. (a) Who did the witness claim/believe (that) the robber attacked?

(b) The historian claimed the king was well respected.

19. (a) Who did the boss guess/assume (that) his employees complained about?

(b) The woman guessed her husband wanted to quit his job.

20. (a) Who did the student suspect/fear (that) her roommate would invite to the party?

(b) The author feared his new book would not sell well.

21. (a) Who did the father fear/suspect (that) his son distrusted?

(b) The farmer suspected the florist was already married.

22. (a) Who did the editor argue/say (that) the journalist should interview?

(b) The union leader argued the employees should be paid more.

23. (a) Who did the king announce/imply (that) the prince married?

(b) The president announced the employees should be paid more. 
24. (a) Who did the prosecutor claim/assume (that) the man robbed?

(b) The midwife assumed the baby was a boy.

Prime and target sentences used in Experiment 3.

1. (a) Who did the manager imply/announce (that) he would promote?

(b) Who did the director announce that he nominated?

2. (a) Who did the activist say/argue (that) that the politician should listen to?

(b) Who did the student say that the teacher scolded?

3. (a) Who did the interviewer believe/suspect (that) the celebrity liked?

(b) Who did the breeder believe that the dog bit?

4. (a) Who did the detective guess/hear (that) the victim saw?

(b) Who did the zookeeper hear that the lion attacked?

5. (a) Who did the man announce/claim (that) he married?

(b) Who did the nurse claim that the patient trusted?

6. (a) Who did the scientist think/claim (that) the politician should consult?

(b) Who did the detective assert that the man robbed?

7. (a) Who did the lawyer suspect/fear (that) her client lied to?

(b) Who did the policeman suspect that the conman tricked?

8. (a) Who did the teacher assume/fear (that) the student hated?

(b) Who did the chef fear that the waiter upset?

9. (a) Who did the journalist hear/guess (that) the politician lied to?

(b) Who did the girl guess that her friend liked?

10. (a) Who did the attorney argue/believe (that) the witness saw? 
(b) Who did the professor argue that the student should listen to?

11. (a) Who did the boy assume/say (that) his sister loved?

(b) Who did the parent assume that the teacher applauded?

12. (a) Who did the fortune teller think/imply (that) the man would marry?

(b) Who did the novelist imply that the main character loved?

13. (a) Who did the mayor announce/imply (that) he would appoint?

(b) Who did the manager imply she would lay off?

14. (a) Who did the activist believe/argue (that) the journalist should criticize?

(b) Who did the girl believe the librarian saw?

15. (a) Who did the zookeeper say/think (that) the penguin loved?

(b) Who did the scientist say she admired?

16. (a) Who did the counselor hear/think (that) the client feared?

(b) Who did the fireman think the boy rescued?

17. (a) Who did the queen guess/hear (that) the knight protected?

(b) Who did the doctor hear the patient was angry at?

18. (a) Who did the witness claim/believe (that) the robber attacked?

(b) Who did the historian claim the king respected?

19. (a) Who did the boss guess/assume (that) his employees complained about?

(b) Who did the woman guess her husband helped?

20. (a) Who did the student suspect/fear (that) her roommate would invite to the party?

(b) Who did the spy fear the commander talked to? 
21. (a) Who did the father fear/suspect (that) his son distrusted?

(b) Who did the farmer suspect the florist married?

22. (a) Who did the editor argue/say (that) that the journalist should interview?

(b) Who did the union leader argue the company treated unfairly?

23. (a) Who did the king announce/imply (that) the prince married?

(b) Who did the president announce he pardoned?

24. (a) Who did the prosecutor claim/assume (that) the man robbed?

(b) Who did veterinarian assume the cat scratched?

Prime and target sentences used in Experiment 4.

1. (a) Who implied/announced (that) he would promote the manager?

(b) Who did the director announce that he nominated?

2. (a) Who argued/said (that) the politician should listen to the activist?

(b) Who did the student say that the teacher scolded?

3. (a) Who suspected/believed (that) the celebrity liked the interviewer?

(b) Who did the breeder believe that the dog bit?

4. (a) Who guessed/heard (that) the victim saw the detective?

(b) Who did the zookeeper hear that the lion attacked?

5. (a) Who announced/claimed (that) she married the actor?

(b) Who did the nurse claim that the patient trusted?

6. (a) Who claimed/thought (that) the politician should consult the scientist?

(b) Who did the detective assert that the man robbed?

7. (a) Who feared/suspected (that) the client lied to the prosecutor? 
(b) Who did the policeman suspect that the conman tricked?

8. (a) Who assumed/feared (that) the student hated the teacher?

(b) Who did the chef fear that the waiter upset?

9. (a) Who heard/guessed (that) the politician lied to the journalist?

(b) Who did the girl guess that her friend liked?

10. (a) Who believed/argued (that) the witness saw the attorney?

(b) Who did the professor argue that the student should listen to?

11. (a) Who said/assumed (that) the boy loved his sister?

(b) Who did the parent assume that the teacher applauded?

12. (a) Who thought/implied (that) the man would marry his childhood friend?

(b) Who did the novelist imply that the main character loved?

13. (a) Who announced/implied (that) the mayor would appoint him?

(b) Who did the manager imply she would lay off?

14. (a) Who argued/believed (that) the activist should criticize the journalist?

(b) Who did the girl believe the librarian saw?

15. (a) Who thought/said (that) the zookeeper loved the penguin?

(b) Who did the scientist say she admired?

16. (a) Who heard/thought (that) the client feared the counselor?

(b) Who did the fireman think the boy rescued?

17. (a) Who guessed/heard (that) the knight protected the queen?

(b) Who did the doctor hear the patient was angry at?

18. (a) Who believed/claimed (that) the robber attacked the pedestrian? 
(b) Who did the historian claim the king respected?

19. (a) Who assumed/guessed (that) his employees complained about the boss?

(b) Who did the woman guess her husband helped?

20. (a) Who suspected/feared (that) the student would invite her roommate to the party?

(b) Who did the spy fear the commander talked to?

21. (a) Who feared/suspected (that) the father distrusted his son?

(b) Who did the farmer suspect the florist married?

22. (a) Who said/argued (that) the journalist should interview the philosopher?

(b) Who did the union leader argue the company treated unfairly?

23. (a) Who implied/announced (that) the princess would marry the peasant?

(b) Who did the president announce he pardoned?

24. (a) Who claimed/assumed (that) the man robbed the bank?

(b) Who did veterinarian assume the cat scratched?

Prime and target sentences used in Experiment 5.

1. (a) The manager implied/announced (that) he would promote them.

(b) I wonder who the director announced that he would nominate.

2. (a) The activist argued/said (that) the politician should listen to them.

(b) I wonder who the student said that the teacher favored.

3. (a) The interviewer suspected/believed (that) the celebrity liked her.

(b) I wonder who the breeder believed that the dog bit. 
4. (a) The detective guessed/heard (that) the victim saw the suspect.

(b) I wonder who the zookeeper heard that the lion attacked.

5. (a) The man announced/claimed (that) he married the actress.

(b) I wonder who the nurse claimed that the patient trusted.

6. (a) The scientist claimed/thought (that) the politician should consult him.

(b) I wonder who the detective thought that the man robbed.

7. (a) The lawyer feared/suspected (that) her client lied to her.

(b) I wonder who the policeman suspected that the conman tricked.

8. (a) The teacher assumed/feared (that) the student hated him.

(b) I wonder who the chef feared that the waiter upset.

9. (a) The journalist heard/guessed (that) the politician lied to him.

(b) I wonder who the girl guessed that her friend liked.

10. (a) The attorney believed/argued (that) the witness lied to the judge.

(b) I wonder who the professor argued that the student should listen to.

11. (a) The boy said/assumed (that) his sister loved his friend.

(b) I wonder who the parent assumed that the teacher applauded.

12. (a) The fortune teller thought/implied (that) the man would marry his old friend.

(b) I wonder wo the novelist implied that the main character loved.

13. (a) The mayor announced/implied (that) he would appoint the expert.

(b) I wonder who the manager implied she would lay off.

14. (a) The activist argued/believed (that) the journalist should criticize the senator. 
(b) I wonder who the librarian believed the book was about.

15. (a) The zookeeper thought/said (that) the penguin loved her.

(b) I wonder who the scientist said she admired.

16. (a) The counselor heard/thought (that) the client feared his brother.

(b) I wonder who the fireman thought the boy rescued.

17. (a) The queen guessed/heard (that) the knight protected the prince.

(b) I wonder who the doctor heard the patient was angry at.

18. (a) The witness believed/claimed (that) the robber attacked the cashier.

(b) I wonder who the historian claimed the king loved.

19. (a) The boss assumed/guessed (that) his employees liked the work environment.

(b) I wonder who the woman guessed her husband helped.

20. (a) The student suspected/feared (that) her roommate would invite him to the party.

(b) I wonder who the spy feared the soldier talked to.

21. (a) The mother feared/suspected (that) her daughter distrusted her.

(b) I wonder who the farmer suspected the florist married.

22. (a) The editor said/argued (that) the journalist should interview them.

(b) I wonder who the union leader argued the company treated unfairly.

23. (a) The king implied/announced (that) the prince would marry the villager.

(b) I wonder who the president announced he pardoned.

24. (a) The prosecutor claimed/assumed (that) the man robbed the old lady.

(b) I wonder who the veterinarian assumed the cat scratched. 
Aoshima, S., Phillips, C., \& Weinberg, A. (2004). Processing filler-gap dependencies in a head-final language. Journal of Memory and Language, 51, $23-54$.

Barr, D. J., Levy, R., Scheepers, C., \& Tily, H. J. (2013). Random effects structure for confirmatory hypothesis testing: Keep it maximal. Journal of Memory and Language, 68, 255-278.

Bernolet, S., \& Hartsuiker, R. J. (2010). Does verb bias modulate syntactic priming? Cognition, 114, 455-461.

Bernolet, S., Hartsuiker, R. J., \& Pickering, M. J. (2012). Effects of phonological feedback on the selection of syntax: Evidence from between-language syntactic priming. Bilingualism: Language and Cognition, 15, 503-516.

Bernolet, S., Hartsuiker, R. J., \& Pickering, M. J. (2013). From languagespecific to shared syntactic representations: The influence of second language proficiency on syntactic sharing in bilinguals. Cognition, 127, 287-306.

Bianchi, V., \& Frascarelli, M. (2017). Complementizer deletion. In M. Everaert, \& H. V. Riemdijk (Eds.), The Blackwell companion to syntax (2nd ed.) (pp. 1-30). Oxford, UK: Blackwell.

Bock, K. (1986). Syntactic persistence in language production. Cognitive Psychology, 18, 355-387.

Bock, K. (1989). Closed-class immanence in sentence production. Cognition, $31,163-186$.

Bock, K., Dell, G. S., Chang, F., \& Onishi, K. H. (2007). Persistent structural priming from language comprehension to language production. Cognition, 104, 437-458.

Bock, K., \& Ferreira, V. S. (2013). Syntactically speaking. In V. S. Ferreira, M. Goldrick, \& M. Miozzo (Eds.), The Oxford handbook of language production (pp. 21-46). Oxford, UK: Oxford University Press. 
Bock, K., \& Griffin, Z. M. (2000). The persistence of structural priming: Transient activation or implicit learning? Journal of Experimental Psychology: General, 129, 177.

Bock, K., Irwin, D. E., Davidson, D. J., \& Levelt, W. J. (2003). Minding the clock. Journal of Memory and Language, 48, 653-685.

Bock, K., \& Levelt, W. J. (1994). Language production: Grammatical encoding. In M. Gernsbacher (Ed.), Handbook of psycholinguistics (pp. 945-984). San Diego, CA: Academic Press.

Bruening, B. (2001). QR obeys superiority: Frozen scope and acd. Linguistic Inquiry, 32, 233-273.

Bürkner, P.-C. (2018). Advanced bayesian multilevel modeling with the r package brms. The $R$ Journal, 10, 395-411.

Cai, Z. G., Pickering, M. J., \& Branigan, H. P. (2012). Mapping concepts to syntax: Evidence from structural priming in mandarin chinese. Journal of Memory and Language, 66, 833-849.

Carminati, M. N., van Gompel, R. P., \& Wakeford, L. J. (2019). An investigation into the lexical boost with nonhead nouns. Journal of Memory and Language, $108,104031$.

Chacón, D. A. (2015). Comparative psychosyntax. Ph.D. thesis University of Maryland, College Park.

Chang, F., Baumann, M., Pappert, S., \& Fitz, H. (2015). Do lemmas speak German? a verb position effect in German structural priming. Cognitive Science, 39, 1113-1130.

Chang, F., Bock, K., \& Goldberg, A. E. (2003). Can thematic roles leave traces of their places? Cognition, 90, 29-49.

Chang, F., Dell, G. S., \& Bock, K. (2006). Becoming syntactic. Psychological Review, 113, 234. 
Chen, X., \& Hartsuiker, R. J. (2021). Do particle verbs share a representation with their root verbs? evidence from structural priming. Journal of Memory and Language, 120, 104255.

Chomsky, N. (1957). Syntactic structure. The Hague: Morton.

Chomsky, N. (1965). Aspects of the theory of syntax. Cambridge, MA: MIT Press.

Chomsky, N. (1973). Conditions on transformations. In S. Anderson, \& P. Kiparsky (Eds.), A festschrift for Morris Halle (pp. 232-286). New York, NY: Holt, Rinehart \& Winston.

Chomsky, N. (1981). Lectures on government and binding: The Pisa lectures. Dordrecht: Foris Publications.

Chomsky, N. (1986). Barriers. Cambridge, MA: MIT press.

Chomsky, N. (1995). The Minimalist program. Cambridge, MA: MIT Press.

Cleland, A. A., \& Pickering, M. J. (2003). The use of lexical and syntactic information in language production: Evidence from the priming of nounphrase structure. Journal of Memory and Language, 49, 214-230.

Clifton, C., \& Frazier, L. (1989). Comprehending sentences with long-distance dependencies. In M. Tanenhaus, \& G. Carlson (Eds.), Linguistic structure in language processing (pp. 273-317). Dortrecht, The Netherlands: Kluwer.

Collins, A. M., \& Loftus, E. F. (1975). A spreading-activation theory of semantic processing. Psychological Review, 82, 407.

Cutting, J. C., \& Ferreira, V. S. (1999). Semantic and phonological information flow in the production lexicon. Journal of Experimental Psychology: Learning, Memory, and Cognition, 25, 318.

Dell, G. S. (1986). A spreading-activation theory of retrieval in sentence production. Psychological Review, 93, 283. 
Dell, G. S., Oppenheim, G. M., \& Kittredge, A. K. (2008). Saying the right word at the right time: Syntagmatic and paradigmatic interference in sentence production. Language and Cognitive Processes, 23, 583-608.

Ferreira, F. (2000). Syntax in language production: An approach using treeadjoining grammars. In L. Wheeldon (Ed.), Aspects of language production (pp. 291-330). San Diego, CA: Psychology press.

Ferreira, V. S. (1996). Is it better to give than to donate? syntactic flexibility in language production. Journal of Memory and Language, 35, 724-755.

Ferreira, V. S. (2003). The persistence of optional complementizer production: Why saying that is not saying that at all. Journal of Memory and Language, $48,379-398$.

Ferreira, V. S., Morgan, A., \& Slevc, L. (2018). Grammatical encoding. In The Oxford handbook of psycholinguistics (2nd ed.) (pp. 432-457). Oxford, UK: Oxford University Press.

Fodor, J. D. (1978). Parsing strategies and constraints on transformations. Linguistic Inquiry, 9, 427-473.

Frank, R. (2004). Phrase structure composition and syntactic dependencies. Cambridge, MA: Mit Press.

Frazier, L., Clifton, C., \& Randall, J. (1983). Filling gaps: Decision principles and structure in sentence comprehension. Cognition, 13, 187-222.

Frazier, L., \& Flores d'Arcais, G. B. (1989). Filler driven parsing: A study of gap filling in dutch. Journal of Memory and Language, 28, 331-344.

Gahl, S., Jurafsky, D., \& Roland, D. (2004). Verb subcategorization frequencies: American English corpus data, methodological studies, and cross-corpus comparisons. Behavior Research Methods, Instruments, $\&$ Computers, 36, $432-443$. 
Garnsey, S. M., Pearlmutter, N. J., Myers, E., \& Lotocky, M. A. (1997). The contributions of verb bias and plausibility to the comprehension of temporarily ambiguous sentences. Journal of Memory and Language, 37, 58-93.

Garnsey, S. M., Tanenhaus, M. K., \& Chapman, R. M. (1989). Evoked potentials and the study of sentence comprehension. Journal of Psycholinguistic Research, 18, 51-60.

van Gompel, R. P., Wakeford, L. J., \& Kantola, L. (2022). No looking back: the effects of visual cues on the lexical boost in structural priming. Language, Cognition and Neuroscience, (pp. 1-10).

Griffin, Z. M., \& Ferreira, V. S. (2006). Properties of spoken language production. In M. Gernsbacher, \& M. Traxler (Eds.), Handbook of psycholinguistics (pp. 21-59). San Diego, CA: Academic Press.

Griffin, Z. M., \& Weinstein-Tull, J. (2003). Conceptual structure modulates structural priming in the production of complex sentences. Journal of Memory and Language, 49, 537-555.

Gruber, J. S. (1965). Studies in lexical relations.. Ph.D. thesis Massachusetts Institute of Technology.

Hall, M. L., Ferreira, V. S., \& Mayberry, R. I. (2015). Syntactic priming in american sign language. PloS one, 10, e0119611.

Hartsuiker, R. J., Bernolet, S., Schoonbaert, S., Speybroeck, S., \& Vanderelst, D. (2008). Syntactic priming persists while the lexical boost decays: Evidence from written and spoken dialogue. Journal of Memory and Language, 58, $214-238$.

Hartsuiker, R. J., \& Kolk, H. H. (1998). Syntactic persistence in Dutch. Language and Speech, 41, 143-184.

Hwang, H., \& Kaiser, E. (2014). Having a syntactic choice is not always better: the effects of syntactic flexibility on Korean production. Language, Cognition and Neuroscience, 29, 1115-1131. 
Jackendoff, R. S. (1985). Semantics and cognition. Cambridge, MA: MIT press.

Jacobs, C. L., Cho, S.-J., \& Watson, D. G. (2019). Self-priming in production: Evidence for a hybrid model of syntactic priming. Cognitive Science, 43 , e12749.

Jaeger, T. F., \& Snider, N. E. (2013). Alignment as a consequence of expectation adaptation: Syntactic priming is affected by the primes prediction error given both prior and recent experience. Cognition, 127, 57-83.

Joshi, A., Levy, L. S., \& Takahashi, M. (1975). Tree adjunct grammars. Journal of Computer and System Sciences, 10, 136-163.

Joshi, A., \& Schabes, Y. (1997). Tree-adjoining grammars. In G. Rozenberg, \& A. Salomaa (Eds.), Handbook of formal languages (pp. 69-123). Berlin, Germany: Springer-Varlag.

Kaschak, M. P., \& Borreggine, K. L. (2008). Is long-term structural priming affected by patterns of experience with individual verbs? Journal of Memory and Language, 58, 862-878.

Kempen, G., \& Hoenkamp, E. (1987). An incremental procedural grammar for sentence formulation. Cognitive Science, 11, 201-258.

Konopka, A. E., \& Bock, K. (2009). Lexical or syntactic control of sentence formulation? structural generalizations from idiom production. Cognitive Psychology, 58, 68-101.

Kratzer, A. (1996). Severing the external argument from its verb. In J. Rooryck, \& L. Zarin (Eds.), Phrase structure and the lexicon (pp. 109-137). Dordrecht, the Netherlands: Springer.

Kroch, A. S., \& Joshi, A. (1985). The linguistic relevance of Tree Adjoining Grammar. Technical Reports (CIS). Department of Computer and Information Science, University of Pennsylvania, (p. 671). 
Larson, R. K. (2017). On dative idioms in english. Linguistic Inquiry, 48, $389-426$.

Lee, E.-K., Brown-Schmidt, S., \& Watson, D. G. (2013). Ways of looking ahead: Hierarchical planning in language production. Cognition, 129, 544-562.

Levelt, W. J. (1989). Speaking: From intention to articulation. Cambridge, MA: MIT press.

Levelt, W. J., \& Kelter, S. (1982). Surface form and memory in question answering. Cognitive Psychology, 14, 78-106.

Levelt, W. J., Roelofs, A., \& Meyer, A. S. (1999). A theory of lexical access in speech production. Behavioral and Brain Sciences, 22, 1-38.

Levin, B., \& Rappaport Hovav, M. (2005). Argument realization. Cambridge, UK: Cambridge University Press.

Lewandowski, D., Kurowicka, D., \& Joe, H. (2009). Generating random correlation matrices based on vines and extended onion method. Journal of Multivariate Analysis, 100, 1989-2001.

Mahon, B. Z., Costa, A., Peterson, R., Vargas, K. A., \& Caramazza, A. (2007). Lexical selection is not by competition: a reinterpretation of semantic interference and facilitation effects in the picture-word interference paradigm. Journal of Experimental Psychology: Learning, Memory, and Cognition, 33, 503.

Mahowald, K., James, A., Futrell, R., \& Gibson, E. (2016). A meta-analysis of syntactic priming in language production. Journal of Memory and Language, $91,5-27$.

Marantz, A. (1993). Implications of asymmetries in double object constructions. In S. Muchombo (Ed.), Theoretical aspects of Bantu grammar (pp. 113-150). Stanford, CA: CSLI Publications. 
McDaniel, D., Cowart, W., McKee, C., \& Garrett, M. F. (2015). The role of the language production system in shaping grammars. Language, (pp. 415-441).

McDonald, J. L., Bock, K., \& Kelly, M. H. (1993). Word and world order: Semantic, phonological, and metrical determinants of serial position. Cognitive Psychology, 25, 188-230.

Melinger, A., \& Dobel, C. (2005). Lexically-driven syntactic priming. Cognition, 98, B11-B20.

Messenger, K., Branigan, H. P., McLean, J. F., \& Sorace, A. (2012). Is young childrens passive syntax semantically constrained? evidence from syntactic priming. Journal of Memory and Language, 66, 568-587.

Momma, S. (2021). Filling the gap in gap-filling: Long-distance dependency formation in sentence production. Cognitive Psychology, 129, 101411.

Momma, S., \& Phillips, C. (2018). The relationship between parsing and generation. Annual Review of Linguistics, 4, 233-254.

Myachykov, A., Scheepers, C., Garrod, S., Thompson, D., \& Fedorova, O. (2013). Syntactic flexibility and competition in sentence production: The case of English and Russian. Quarterly Journal of Experimental Psychology, $66,1601-1619$.

Navarrete, E., Del Prato, P., Peressotti, F., \& Mahon, B. Z. (2014). Lexical selection is not by competition: Evidence from the blocked naming paradigm. Journal of Memory and Language, 76, 253-272.

Omaki, A., Lau, E. F., Davidson White, I., Dakan, M. L., Apple, A., \& Phillips, C. (2015). Hyper-active gap filling. Frontiers in Psychology, 6, 384.

Pesetsky, D. (2017). Complementizer-trace effects. In M. Everaert, \& H. van Riemsdijk (Eds.), The Blackwell companion to syntax (2nd ed.). Malden, MA: Willy-Blackwell. 
Phillips, C. (2013). On the nature of island constraints II: Language learning and innateness. In J. Sprouse, \& N. Hornstein (Eds.), Experimental syntax and island effects (pp. 132-158). Cambridge, UK: Cambridge University Press.

Pickering, M. J., \& Branigan, H. P. (1998). The representation of verbs: Evidence from syntactic priming in language production. Journal of Memory and Language, 39, 633-651.

Pickering, M. J., \& Branigan, H. P. (2019). Special issue on structural priming in less-studied languages and dialects: Introduction. Journal of Cultural Cognitive Science, 3, 1-4.

Pickering, M. J., \& Ferreira, V. S. (2008). Structural priming: a critical review. Psychological Bulletin, 134, 427.

Pollard, C., \& Sag, I. A. (1994). Head-driven Phrase Structure Grammar. Chicago, IL: University of Chicago Press.

Potter, M. C., \& Lombardi, L. (1990). Regeneration in the short-term recall of sentences. Journal of Memory and Language, 29, 633-654.

Potter, M. C., \& Lombardi, L. (1998). Syntactic priming in immediate recall of sentences. Journal of Memory and Language, 38, 265-282.

Pylkkänen, L. (2008). Introducing arguments. Cambridge, MA: MIT press.

R Core Team (2020). R: A Language and Environment for Statistical Computing. R Foundation for Statistical Computing Vienna, Austria. URL: https://www.R-project.org/.

Reitter, D., Keller, F., \& Moore, J. D. (2011). A computational cognitive model of syntactic priming. Cognitive Science, 35, 587-637.

Roelofs, A. (1992). A spreading-activation theory of lemma retrieval in speaking. Cognition, 42, 107-142. 
Roelofs, A. (1997). The weaver model of word-form encoding in speech production. Cognition, 64, 249-284.

Roland, D., Elman, J. L., \& Ferreira, V. S. (2006). Why is that? structural prediction and ambiguity resolution in a very large corpus of english sentences. Cognition, 98, 245-272.

Ross, J. (1967). Constraints on variables in syntax.. Ph.D. thesis Yale University.

Schad, D. J., Vasishth, S., Hohenstein, S., \& Kliegl, R. (2020). How to capitalize on a priori contrasts in linear (mixed) models: A tutorial. Journal of Memory and Language, 110, 104038.

Scheepers, C. (2003). Syntactic priming of relative clause attachments: Persistence of structural configuration in sentence production. Cognition, 89, 179-205.

Scheepers, C., Raffray, C. N., \& Myachykov, A. (2017). The lexical boost effect is not diagnostic of lexically-specific syntactic representations. Journal of Memory and Language, 95, 102-115.

Segaert, K., Menenti, L., Weber, K., \& Hagoort, P. (2011). A paradox of syntactic priming: why response tendencies show priming for passives, and response latencies show priming for actives. PloS one, 6, e24209.

Sprenger, S. A., Levelt, W. J., \& Kempen, G. (2006). Lexical access during the production of idiomatic phrases. Journal of Memory and Language, 54, $161-184$.

Stowe, L. A. (1986). Parsing wh-constructions: Evidence for on-line gap location. Language and Cognitive Processes, 1, 227-245.

Stowell, T. A. (1981). Origins of phrase structure. Ph.D. thesis Massachusetts Institute of Technology. 
Vasishth, S., Nicenboim, B., Beckman, M. E., Li, F., \& Kong, E. J. (2018). Bayesian data analysis in the phonetic sciences: A tutorial introduction. Journal of Phonetics, 71, 147-161.

Van de Velde, M., Kempen, G., \& Harbusch, K. (2015). Dative alternation and planning scope in spoken language: A corpus study on effects of verb bias in vo and ov clauses of dutch. Lingua, 165, 92-108.

Vijay-Shanker, K. (1992). Using descriptions of trees in a Tree Adjoining Grammar. Computational Linguistics, 18, 481-518.

Wanner, H., \& Maratsos, M. (1978). An ATN approach to comprehension. In Linguistic theory and psychological reality. Cambridge, MA: MIT Press.

Yan, H., Martin, R. C., \& Slevc, L. R. (2018). Lexical overlap increases syntactic priming in aphasia independently of short-term memory abilities: Evidence against the explicit memory account of the lexical boost. Journal of Neurolinguistics, 48, 76-89.

Zehr, J., \& Schwarz, F. (2018). PennController for internet based experiments (IBEX). https://doi.org/10.17605/OSF.IO/MD832.

Ziegler, J., Bencini, G., Goldberg, A., \& Snedeker, J. (2019). How abstract is syntax? evidence from structural priming. Cognition, 193, 104045. 